\title{
Some Probabilistic and Statistical Properties of the Seismic Regime of Zemmouri (Algeria) Seismoactive Zone
}

\author{
Kamel BADDARI ${ }^{1,3,4}$, Fouzi BELLALEM ${ }^{2}$, Ibtihel BADDARI ${ }^{3}$, \\ and Said MAKDECHE ${ }^{4}$
}

${ }^{1}$ Lab of Physics of the Earth, Université Mhamed Bougara Boumerdes, Algeria

${ }^{2}$ Centre de Recherche en Astronomie, Astrophysique et Géophysique du Globe, Algiers, Algeria

${ }^{3}$ Laboratoire d'Informatique, de Modélisation, d'Optimisation et des Systèmes Électroniques, Université Mhamed Bougara Boumerdes, Algeria

${ }^{4}$ University of Bouira, Bouira, Algeria; e-mail: k.badari@mesrs.dz

\begin{abstract}
Statistical tests have been used to adjust the Zemmouri seismic data using a distribution function. The Pareto law has been used and the probabilities of various expected earthquakes were computed. A mathematical expression giving the quantiles was established. The extreme values limiting law confirmed the accuracy of the adjustment method. Using the moment magnitude scale, a probabilistic model was made to predict the occurrences of strong earthquakes. The seismic structure has been characterized by the slope of the recurrence plot $\gamma$, fractal dimension $D$, concentration parameter $K_{s r}$, Hurst exponents $H_{r}$ and $H_{t}$. The values of $D, \gamma$, $K_{s}, H_{r}$, and $H_{t}$ diminished many months before the principal seismic shock $(M=6.9)$ of the studied seismoactive zone has occurred. Three stages of the deformation of the geophysical medium are manifested in the variation of the coefficient $G \%$ of the clustering of minor seismic events.
\end{abstract}

Key words: Pareto law, Fréchet law, earthquake forecasting, seismic regime.

Ownership: Institute of Geophysics, Polish Academy of Sciences;

(C) 2016 Baddari et al. This is an open access article distributed under the Creative Commons Attribution-NonCommercial-NoDerivs license,

http://creativecommons.org/licenses/by-nc-nd/3.0/. 


\section{INTRODUCTION}

The seismic phenomenon is supposed to be the result of a complex stochastic process. Several probabilistic models for earthquake occurrence in different seismoactive zones of the world are currently available (Console et al. 2003, Ogata et al. 2003, Zhuang et al. 2004). Baddari et al. (2013) presented the results of estimation of seismic moment values of a prescribed exceedence probability of the seismoactive zone of Zemmouri in Algeria using the moment magnitude distribution. Physical and statistical models were provided to study the earthquake clustering phenomenon (Kanamori 2004, Aitouche et al. 2013, Baddari et al. 2015). The principal approach in predicting macrofailure in relation to earthquake forecasting was based on the Poisson law (Zavyalov 2006, Baddari et al. 2011). The common property to all these seismic models is that the earthquakes occurrences have a stochastic nature. The Gutenberg-Richter law (GRL) was treated in probabilistic terms to study the seismicity and the structure of Earth's interior (Evison 1999, Lavanda and Cipollone 2000). Therefore, the GRL is regarded to be of high accuracy for large space-time sizes in the diapason of small and moderate earthquake magnitudes. Several methods are used in earthquake science to describe their physical processes. The earthquake, in laboratory experiments, is associated to a process of evolution and concentration of microruptures at various scales to give place finally to a main macrorupture inducing the instability and the destruction of the medium (Sobolev and Ponomarev 2003, Baddari et al. 1999, 2012). The random distribution of the ruptures in the stress field can be approached using the Poisson distribution (Zavyalov 2006, Baddari et al. 2011). Self similarity of seismic regime has been raised by Fukao and Furumento (1985), Crownover (1995), Baddari et al. (1996), Caneva and Smirnov (2004). One of the forms of self similarity can be expressed by the slope of the curve of recurrence and the fractal dimension of the earthquakes' spatial distribution. The evaluation of the statistical parameters defining the seismic regime reflects the evolution of the physical process in the Earth crust.

The present paper suggests the elaboration of probabilistic and statistical characteristics for the Zemmouri seismoactive region based on the adjustment tests, the extreme values limiting law and some statistical parameters of the seismic regime. The extreme values limiting law was applied to demonstrate that the Pareto law belongs to the attraction field of the Fréchet law and to confirm the accuracy of the adjustment method that has been conducted. We present the results of estimation of seismic moment values of a prescribed exceedance probability of the seismoactive zone of Zemmouri in Algeria using the moment magnitude distribution. Once the three distribution functions of these notions are defined, they will be used to calculate the 
probability of exceeding a threshold, which allows us to estimate or not the occurrence of a strong earthquake. Some regularities in seismic regime have been studied during the preparation and development of dynamic events. The time-space behavior of the slope of the recurrence plot $\gamma$, fractal dimension of the hypocenter set $D$, the relationship $D-3 \gamma$, crack concentration parameter $K_{s}$, and the Hurst exponent $H$ in field experiments in Zemmouri seismoactive zone have been analyzed as well.

\section{INPUT DATA AND METHODOLOGY}

We studied the seismic activity extending between $1.8^{\circ}-5^{\circ} \mathrm{E}$ and $34.5^{\circ}$ $37.5^{\circ} \mathrm{N}$ of the Zemmouri area (Northern of Algeria) during the period 19802003. The aftershocks were eliminated from the catalogue. This area is one of the most seismically active regions in the western Mediterranean Sea. The area was a site of a large earthquake on 21 May $2003(M=6.9)$, whose localization was $3.6^{\circ} \mathrm{E}$ and $36.96^{\circ} \mathrm{N}$. To compile the earthquake catalog, we have reported only well-documented events that have been revised by different sources (Benouar 1994, Mokrane et al. 1994, Yelles Chaouche et al. 2002) and extended data from ISC and CSEM bulletins for the most recent events. As in any compilation, some events are reported several times and a sequential elimination of doublets and redundant events was performed. The compiled catalog covers the period 1980-2003 and consists of 320 shallow events with $M \geq 2$. The catalog of main shocks has been compiled from the raw earthquake catalog by eliminating the aftershocks using the windowing technique introduced by Gardner and Knopoff (1974). For each earthquake epicenter, a circular space window and a time window are determined, so that any earthquake occurring within the window is deemed a cluster event. Those are intermediate between the broad range of values of Gardner and Knopoff. The residual catalog includes 70 events with $M \geq 3.4$. Figure 1 shows the epicenter distribution of earthquakes between 1980 and 2003 after declustering. Descriptive statistics of seismicity of the studied area are detailed in Appendix. The auto similarity parameters: $\gamma$-value, fractal dimension $D$, the relation $D-3 \gamma$ and seismogenic concentration parameter $K_{s r}$ have been calculated in replica zones for space of earthquake preparation and including a determined number of seismic events of $M \geq 2$ during the time interval $\Delta T=1.5$ years. Each zone (earthquake preparation area) has been divided into elementary volumes of linear dimension $\Delta X=(5-7) L_{i}$ and depth $\Delta Z=20 \mathrm{~km}$. The area of aftershocks constituted the boundary of the skeiling field. The choice of the elementary volume dimensions and window $\Delta T$ duration depends on the size of a source area of an earthquake to be predicted. 


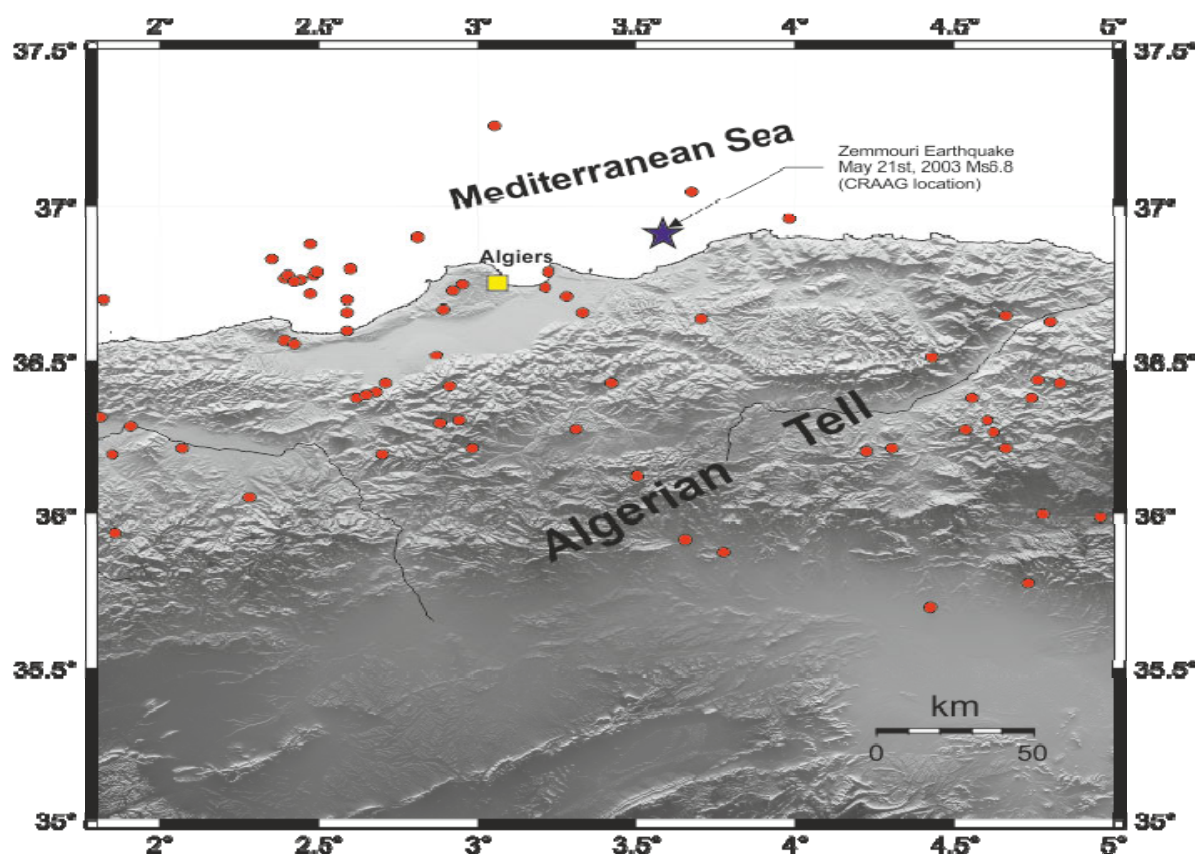

Fig. 1. Seismicity map of the Zemmouri area since 1980 till $2003(m \geq 3.4)$.

\section{MAGNITUDE ADJUSTMENT BY DISTRIBUTION FUNCTION}

\subsection{Adjustment tests}

The sample of 70 seismic events was divided into 9 classes, then reduced to 4 classes, by gathering the 6 last ones in only one class, so that the values of each exceed 5 as the test requires it. It is the $H_{0}$ test (null hypothesis) against $H_{1}$ test (alternative hypothesis)

$$
\begin{cases}H_{0} & \text { if } F(m)=F_{0}(m) \\ H_{1} & \text { if } F(m) \neq F_{0}(m)\end{cases}
$$

where: $F(m)$ indicates the random distribution function of the sampled variable $M$ (magnitude), $F_{0}(m)$ is the suggested usual distribution function and $m$ are the values of the random variable $M$. Initially, the plot histogram of relative frequencies showed that the polygon obtained by Table 1 corresponded to the same shape as the graph of density of the Pareto law. In a second step, the establishment of the cumulative frequencies polygon representing the empirical distribution function (Fig. 2a) showed that it has the same form as the Pareto distribution function (Fig. 2b). So, the $m$ values followed the Pareto law of parameter $\alpha$ and have a density function $f_{0}(m)=$ $(\alpha / 4)(3.4 / m)^{\alpha+1}$ if $m \geq 3.4, f_{0}(m)=0$ else, and a distribution function 
Table 1

Elements of calculation necessary for the $\chi^{2}$ test adjustment

\begin{tabular}{|l|l|r|l|}
\hline Classes & $n_{i}$ & \multicolumn{1}{|c|}{$n p_{i}$} & $\left(n_{i}-n p_{i}\right)^{2} / n p_{i}$ \\
\hline$[3.4,3.8[$ & 24 & 30.96 & 1.5664651163 \\
{$[3.8,4.2[$} & 22 & 15.95 & 2.294827586 \\
{$[4.2,4.6[$} & 14 & 8.76 & 3.134429224 \\
{$[4.6,7[$} & 10 & 12.74 & 0.589293563 \\
\hline Total & $n=70$ & & 7.585015661 \\
\hline
\end{tabular}

Explanations: $k=4$ classes; $\ell=1$ for $\alpha=5.25$.

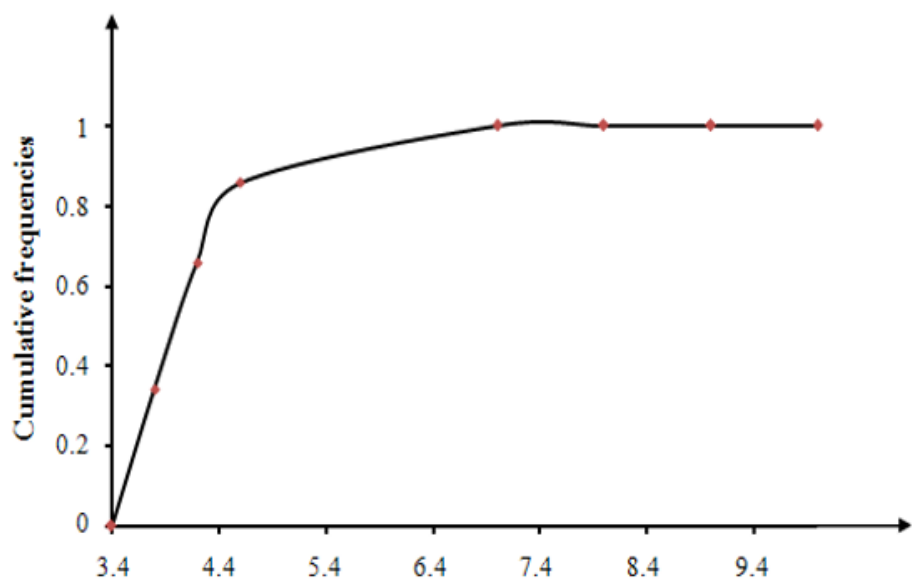

Magnitudes (m)

Fig. 2a. Empirical curve of relative frequencies.

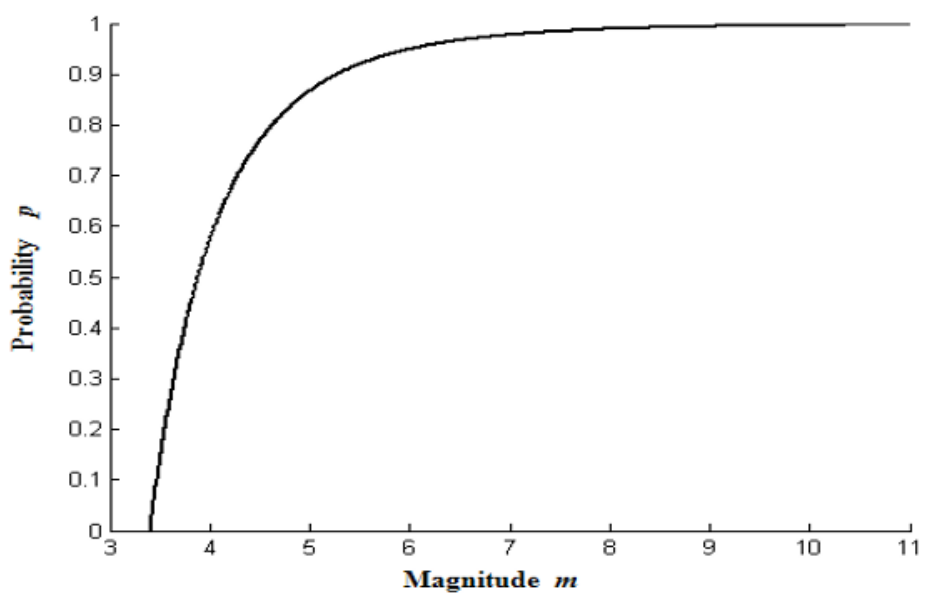

Fig. 2b. Pareto distribution function. 
$F_{0}(m)=1-(3.4 / m)^{\alpha}$, with the Pareto mathematical expectation $E(M)=$ $[\alpha /(\alpha-1)] 3.4=4.2=\bar{M}$, where $\bar{M}$ is the arithmetic mean of the random variable (magnitude) of the selected 70 earthquakes. The estimator $\alpha$ was found to be equal to 5.25. Table 1 shows the elements of calculation necessary for the $\chi^{2}$ test adjustment, which asymptotically followed, when the number of samples $n \rightarrow \infty$, the $\chi^{2}$ law with $(k-1-\ell)$ degrees of freedom.

The null hypothesis

$$
H_{0}=\left(F(m)=F_{0}(m)=1-\left(\frac{3.4}{m}\right)^{5.25}\right)
$$

was accepted with an error of $\alpha_{0}=0.01$, i.e., the probability of $1-\alpha_{0}=$ 0.99 , proposing $k=4$ classes; $\ell=1 ; \alpha=5.25$.

Table 2 shows the KS test with $F(m)=F_{0}(m)$. By comparing $D_{n}=$ $\max \mid F_{n}(m)-F(m)$, which indicates the maximum difference between the empirical distribution function and the Pareto distribution function, and the critical values $d_{n}$ read on the KS table (Kolmogorov 1974), and setting up $\alpha_{0}$, we obtained, for $D_{n}>d_{n}$, the hypothesis $H_{0}\left(P\left(D_{n}>d_{n}\right)=\alpha_{0}\right)$, which was rejected with a risk of error of $\alpha_{0}$. Supposing that $D_{n}=0.099442, n=70$, $\alpha_{0}=0.01$, and $d_{n}=0.1916$, we obtain $D_{n}<0.19167$ and we conclude that the hypothesis $H_{0}$ given by formula 2 should not be rejected with a risk of error of $\alpha_{0}=0.01$, i.e., the probability of $P\left(D_{n}<d_{n}\right)=1-\alpha_{0}=0.99$. According to the KS table, Table 2 shows that $D_{n}=0.099442$ and $d_{n}=0.19167$. For $n=70$ and $\alpha_{0}=20 \%$, we have $d_{n}=0.12586$; for $\alpha_{0}=10 \%$, we have $d_{n}=0.14431 ;$ and for $\alpha_{0}=5 \%$, we have $d_{n}=0.15975$. In all three cases, $D_{n}=0.099442<d_{n}$, and, consequently, the hypothesis $H_{0}$ is not rejected. We conclude that the two tests confirm that the sampled random variable $M$ is adjusted using the Pareto distribution function with a risk of error of 0.01 (a probability of $99 \%$ ), i.e.

Table 2

Elements of calculation necessary for the Kolmogorov-Smirnov test adjustment

\begin{tabular}{|c|c|c|c|c|c|}
\hline Classes & $n_{i}$ & $N_{i}$ & $F_{n}(m)$ & $F_{0}(m)$ & $F_{n}(m)-F_{0}(m)$ \\
\hline$[3.4,3.8[$ & 24 & 24 & 0.342858 & 0.4423000 & 0.0994420 \\
{$[3.8,4.2[$} & 22 & 46 & 0.657143 & 0.6702343 & 0.0130913 \\
{$[4.2,4.6[$} & 14 & 60 & 0.857143 & 0.7954566 & 0.0616864 \\
{$[4.6,7[$} & 10 & 70 & 1.000000 & 0.9774317 & 0.0225683 \\
\hline
\end{tabular}

Explanation: $F_{n}(m)$ is the distribution empirical function. 


$$
F(m)=1-\left(\frac{3.4}{m}\right)^{5.25}
$$

Figure 3 shows the graph of the probability $P\left(M \geq m_{i}\right)=1-F(m)=$ $\bar{F}(m)$. Table 3 gives some probabilities $P\left(M \geq m_{i}\right)$ of the forthcoming earth-

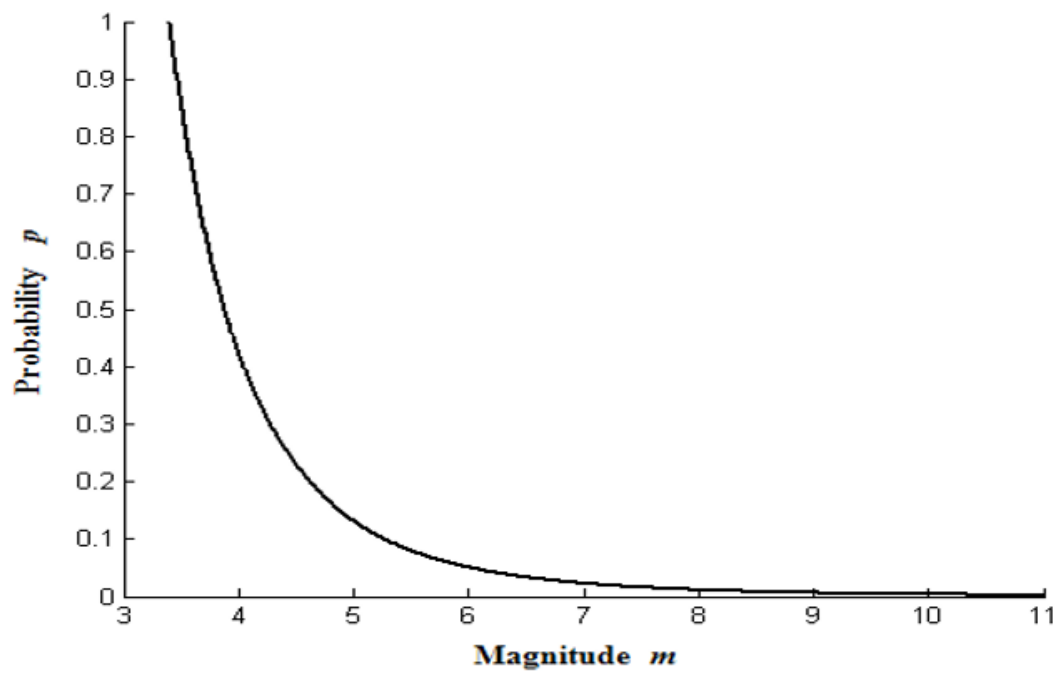

Fig. 3. Graph representation of $P\left(M \geq m_{i}\right)=1-F_{M}(m)=\overline{F_{M}}(m)$.

Table 3

Probabilities of forthcoming earthquakes exceeding a certain magnitude $\left(m_{i}\right)_{i=1 \ldots 9}$ : $5 ; 5.5 ; 6 ; 6.5 ; 7 ; 7.5 ; 8 ; 8.5 ; 9$, and the probabilities of earthquakes for which the magnitude is ranging between $m_{i-1}$ and $m_{i}$ for $i=2$ to $9\left(P\left(m_{\mathrm{i}-1} \leq M \leq m_{i}\right)\right)$

\begin{tabular}{|l|l|c|}
\hline$m_{i}$ & $P\left(M \geq m_{i}\right)$ & $P\left(m_{i-1} \leq M \leq m_{i}\right)$ \\
\hline 5 & 0.13202975 & $P(5 \leq M \leq 5.5)=0.05198$ \\
5.5 & 0.08004979 & $P(5.5 \leq M \leq 6)=0.0293542$ \\
6 & 0.0506956 & $P(6 \leq M \leq 6.5)=0.0173937$ \\
6.5 & 0.0333019 & $P(6.5 \leq M \leq 7)=0.0107336$ \\
7 & 0.02256834 & $P(7 \leq M \leq 7.5)=0.0068578$ \\
7.5 & 0.0157106 & $P(7.5 \leq M \leq 8)=0.00451514$ \\
8 & 0.01119546 & $P(8 \leq M \leq 8.5)=0.0030519$ \\
8.5 & 0.0081436 & $P(8.5 \leq M \leq 9)=0.0021112$ \\
9 & 0.006032414 & \\
\hline
\end{tabular}


quakes exceeding the magnitudes $m_{i}$. To study the probabilistic characteristics based on the distribution function of the magnitude scale, we calculated the quantile $m_{q}$ (the return cycle) of order $q=1-p$ such as $q=F\left(m_{q}\right)$, and we have

$$
m_{q}=\frac{3.4}{(1-q)^{\frac{1}{5.25}}} \approx \frac{3.4}{(1-q)^{0.19}} .
$$

Table 4 gives $m_{q}$ for determined $q$. We have given 14 values of $q$ and we have obtained 14 magnitudes $m_{q}$. As an example, a magnitude of 3.5 corresponds to $q=0.2$ and a magnitude of 8.5 corresponds to $q=0.992$. The probabilistic interpretation of these results is $F(3.5)=P(M \leq 3.5)=0.2$, i.e., $P(M \geq 3.5)=0.8$ and $F(8.5)=P(M \leq 8.5)=0.992$, i.e., $P(M \geq 8.5)=0.008$.

Table 4

Quantiles $m_{q}$ for a given $q$

\begin{tabular}{|c|l|}
\hline Magnitude $m_{q}$ & \multicolumn{1}{|c|}{$q$} \\
\hline 3.5 & 0.2 \\
3.6 & 0.3 \\
3.7 & 0.4 \\
3.9 & 0.5 \\
4 & 0.6 \\
4.3 & 0.7 \\
4.4 & 0.75 \\
4.6 & 0.8 \\
5.3 & 0.9 \\
6 & 0.95 \\
6.3 & 0.96 \\
6.6 & 0.97 \\
8.2 & 0.99 \\
8.5 & 0.992 \\
\hline
\end{tabular}

\subsection{Probability of the expected maximum magnitude of $\boldsymbol{n}$ earthquakes}

We have

$$
\begin{aligned}
& P\left(\max M_{i}>m\right)=1-P\left(\max _{i=1, n} M_{i} \leq m\right)= \\
& 1-P\left(M_{1} \leq m \wedge \ldots . \wedge M_{n} \leq m\right)=1-\prod_{i=1}^{n} P\left(M_{i} \leq m\right)
\end{aligned}
$$


knowing that $M_{i}$ are independent random variables for $m \geq 3.4$, thus

$$
P\left(\max _{i=1, n} M_{i} \geq m\right)=1-\left(1-\left(\frac{3.4}{m}\right)^{5.25}\right)^{n}=\bar{G}(m)=1-G(m)
$$

$G(m)$ is related to the distribution function of the variable $\max _{i=1, n} M_{i}$ and $\bar{G}(m)$ represents $1-G(m)$. Figure 4 shows three graphics of the function $\bar{G}(m)$ for $n=2, n=3$, and $n=4$ earthquakes.

We notice that the probability increases when the sample number $n$ increases. Table 5 gives the probabilities of maximum magnitude of $n$ earthquakes of magnitude $m \geq 3.4$ falling between given magnitudes $a$ and $b$. The probability was calculated by the following formula

$$
P\left(a \leq \max _{i=1, n} M_{i} \leq b\right)=\left(1-\left(\frac{3.4}{b}\right)^{5.25}\right)^{n}-\left(1-\left(\frac{3.4}{a}\right)^{5.25}\right)^{n} .
$$

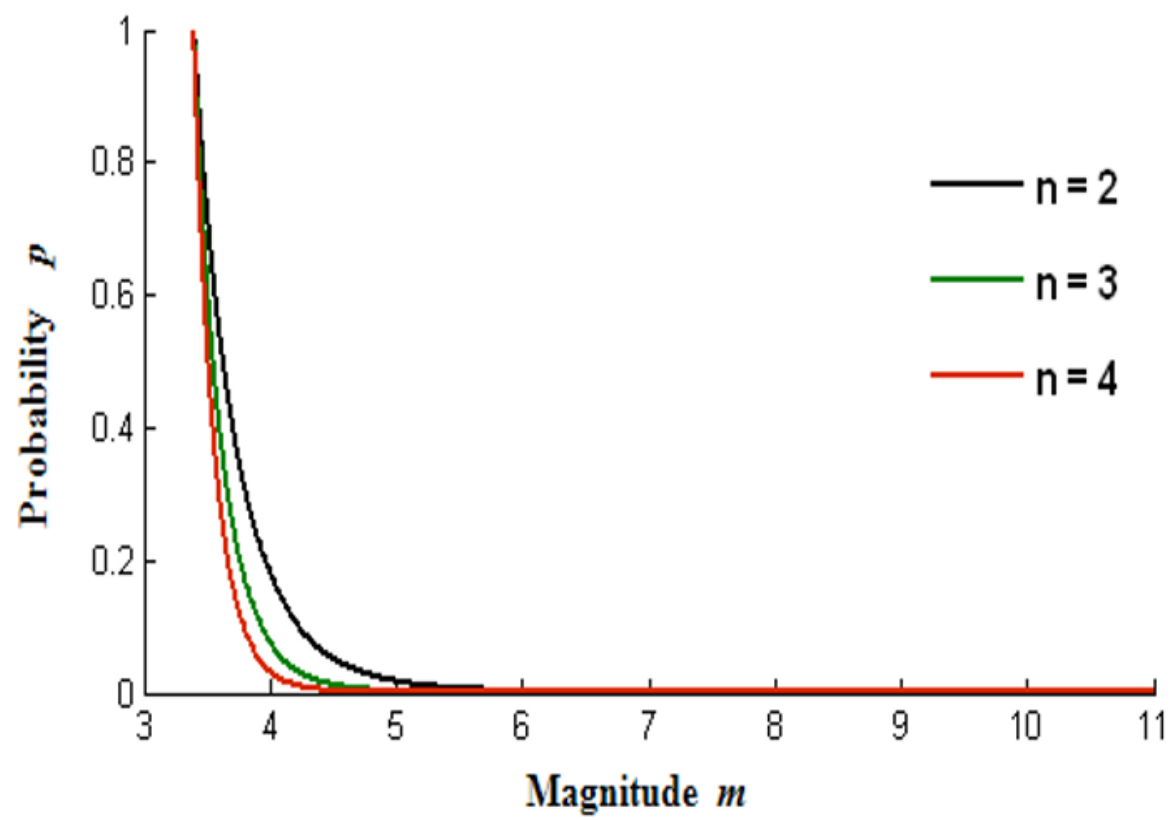

Fig. 4. Graph representation of $P\left(\max _{i=1, n} M_{i} \geq m\right)$ for $n=2, n=3$, and $n=4$; $m \geq 3.4$. 
Table 5

Probabilities of $\max _{j=1, n} M_{j}$ to exceed $m_{i}$ and probabilities of $\max _{j=1, n} M_{j}$ to be included between $m_{i-1}$ and $m_{i}$

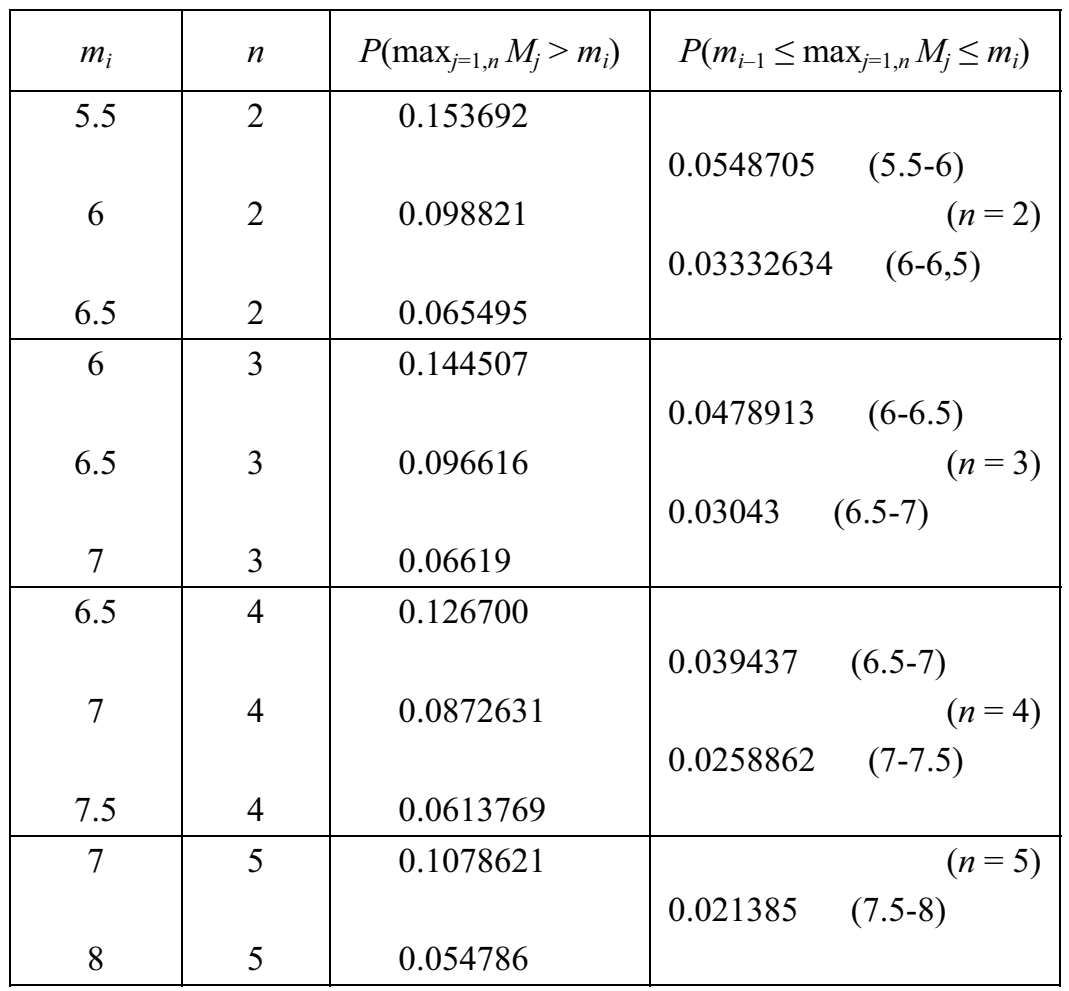

\subsection{Application of the extreme values limiting law}

We found that the Pareto distribution function belongs to the Fréchet attraction field of parameter $\alpha=5.25$, i.e.

$$
\phi_{\alpha}(m)=\left\{\begin{array}{cc}
0 & m \leq 0 \\
\mathrm{e}^{-m^{-5.25}} & m \geq 0
\end{array}\right.
$$

By applying the condition of the Von Mises criterion (De Haan 1990), we obtain

$$
\lim _{x \rightarrow \infty} \frac{m \frac{5.25}{3.4}\left(\frac{3.4}{m}\right)^{6.25}}{\left(\frac{3.4}{m}\right)^{5.25}}=\lim _{x \rightarrow \infty} \frac{5.25\left(\frac{3.4}{m}\right)^{5.25}}{\left(\frac{3.4}{m}\right)^{5.25}}=5.25, \forall m
$$




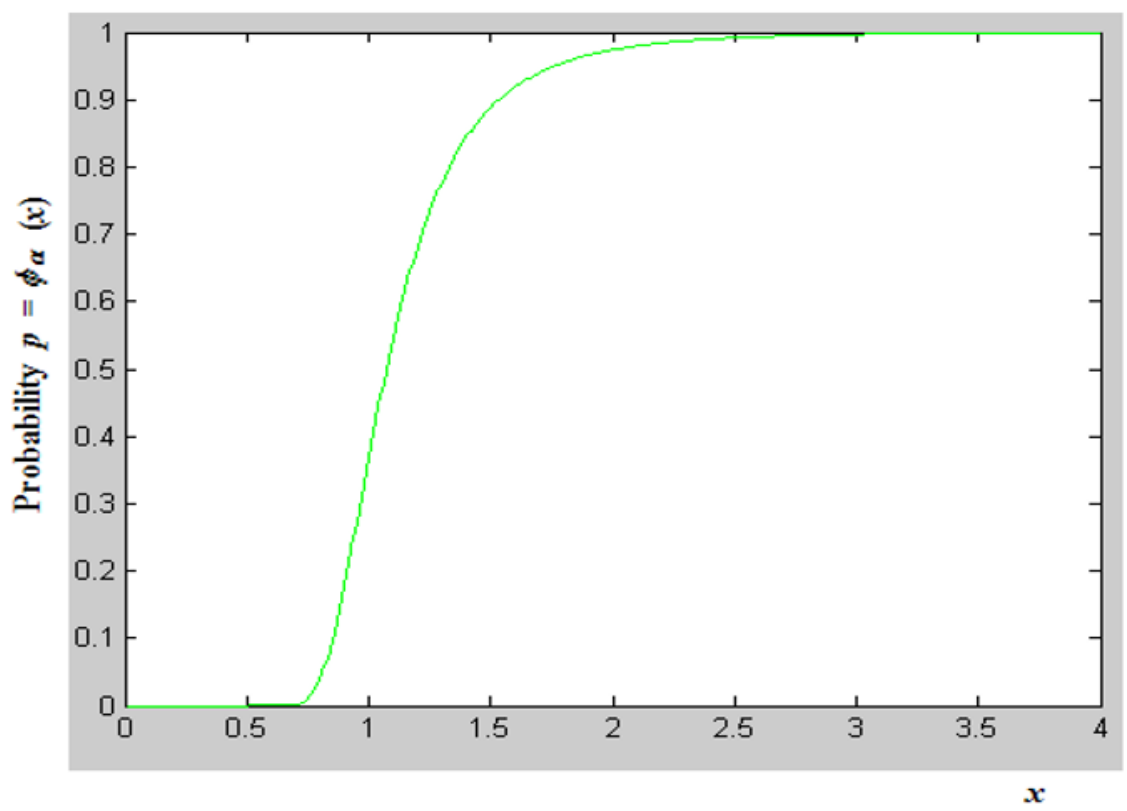

Fig. 5. Graph representation of the distribution function of Fréchet $\phi_{a}(\mathrm{~m})$ of parameter $\alpha=5.25$.

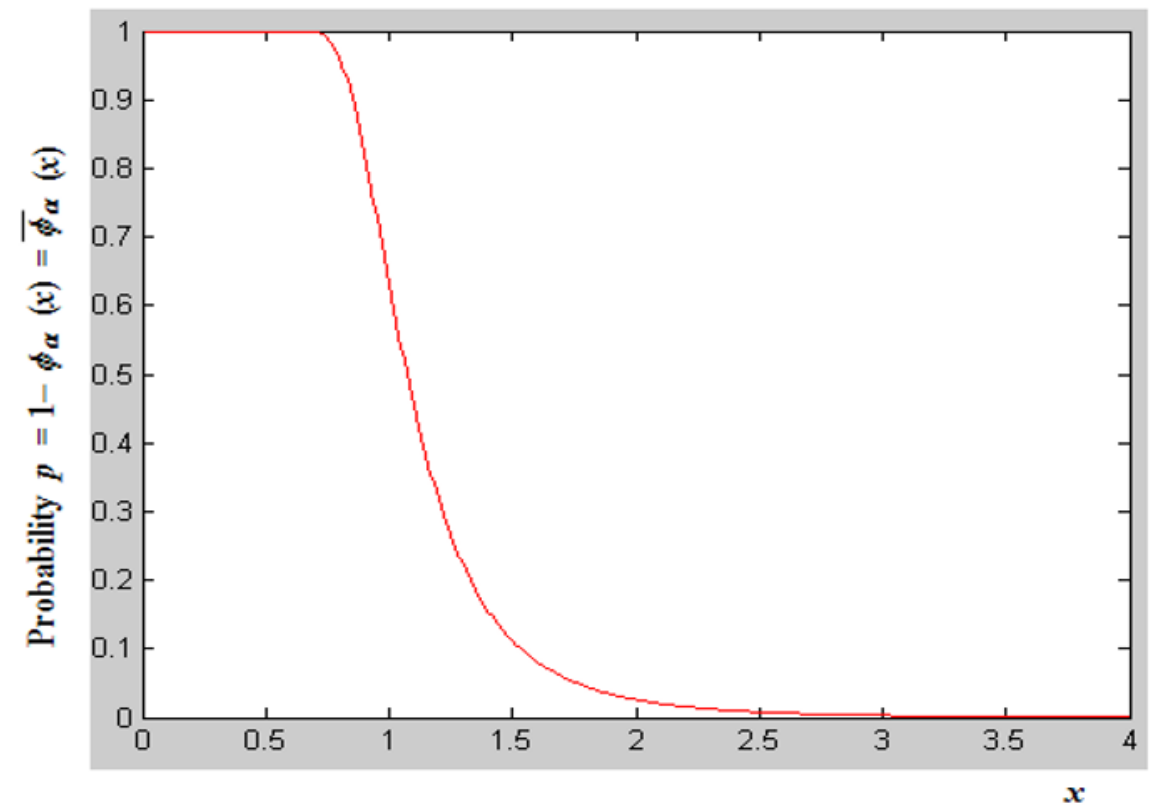

Fig. 6. Graph representation of the function $1-\phi_{\alpha}(m)=\bar{\phi}_{\alpha}(m)$. 
According to the Haan and Ferreira theorem, we can determine the reciprocal function $F^{-1}(\mathrm{~m})$. Figure 5 presents the distribution function of the Fréchet law. This latter allows us to find the maximum probability, as well the probability of having a magnitude less than $x$. Figure 6 shows the representation of the probability $1-\phi_{\alpha}(m)$ so that the maximal magnitude is over the value $x$.

\subsection{Comparison of the probabilities}

When we compare the two following formulas obtained, respectively, by the Pareto law and the extreme values limiting law, we have

$$
\begin{aligned}
& P\left(\max _{i=1, n} M_{i} \geq M_{0}\right)=1-\left(1-\left(\frac{3.4}{M_{0}}\right)^{5.25}\right)^{n} \\
& P\left(\max _{i=1, n} M_{i}>3.4 n^{\frac{1}{5.25}} x\right) \rightarrow 1-\frac{1}{e^{\frac{1}{m^{5.25}}}}
\end{aligned}
$$

Considering that

$$
x=\frac{M_{0}}{(3.4) n^{\frac{1}{5.25}}}
$$

where $M_{0}$ is the given magnitude, we can notice that for $M_{0}=6.9$ and $n \geq 30$, the absolute difference between the probabilities given by formulas 10 and 11 was 0.04 , and the relative error was about $0.7 \%$. For $M_{0}=8$ and $n \geq 30$, the absolute difference was about 0.001 , and the relative error was $0.4 \%$. Thus, we can say that the probability given by formula 10 appears much more interesting compared to that given by formula 11, because with the latter the probability can be calculated only for rather large number $n$ of seismic events. Indeed, with formula 10, the probability can be calculated for any $n$ and it is possible to give the value of $n$ for a given $p$, and we have

$$
n \ln \left(1-\left(\frac{3.4}{M_{0}}\right)^{5.25}\right)=\ln (1-p)
$$

where the number of earthquakes is

$$
n=\left[\frac{\ln (1-p)}{\ln \left(1-\left(\frac{3.4}{M_{0}}\right)^{5.25}\right)}\right]
$$

and [.] indicates the whole part. 


\section{DISTRIBUTION FUNCTIONS OF THE MOMENT MAGNITUDE SCALE}

It is noteworthy that serious constraints affect the magnitude scales in the case of a large local or regional seismic event, such as saturation and discrepancies between scales (Kanamori 1977, Das et al. 2011). The moment magnitude scale is physically trustworthy to predict source parameters and strong motion amplitudes (Utsu 2002). For this reason, we have used the moment magnitude in our model. We used the relation

$$
\log M_{0}=1.5 M_{L}+16.0 \text {. }
$$

To determine the distribution function $G(x)$ of $Y=\log M_{0}$, we put

$$
Y=\log M_{0}=1.5 M_{L}+16
$$

or

$$
Y=1.5 M+16
$$

Knowing that

$$
G(x)=P(Y \leq x)=P(1.5 M+16 \leq x)
$$

we obtain

$$
G(x)=P\left(M \leq \frac{2(x-16)}{3}\right)=F\left(\frac{2(x-16)}{3}\right)
$$

where $F$ is the Pareto distribution function. Since

$$
F\left(\frac{2(x-16)}{3}\right)=\left\{\begin{array}{cc}
1-\left(\frac{3.4}{2(x-16)}\right)^{5.25} & \text { if } \quad \frac{2(x-16)}{3} \geq 3.4 \\
0 & \text { else }
\end{array}\right.
$$

the distribution function $G(x)$ of $Y=\log M_{0}$ is written as

$$
G(x)=\left\{\begin{array}{lcc}
1-\left(\frac{5.1}{x-16}\right)^{5.25} & \text { if } & x \geq 21.1 \\
0 & & \text { else }
\end{array} .\right.
$$

Figure 7 represents the distribution function of the random variable $Y$.

The distribution function $H(y)$ of the random variable $Z_{i}=M_{0 i}$ of the moment of the $i^{m e}$ earthquake is 


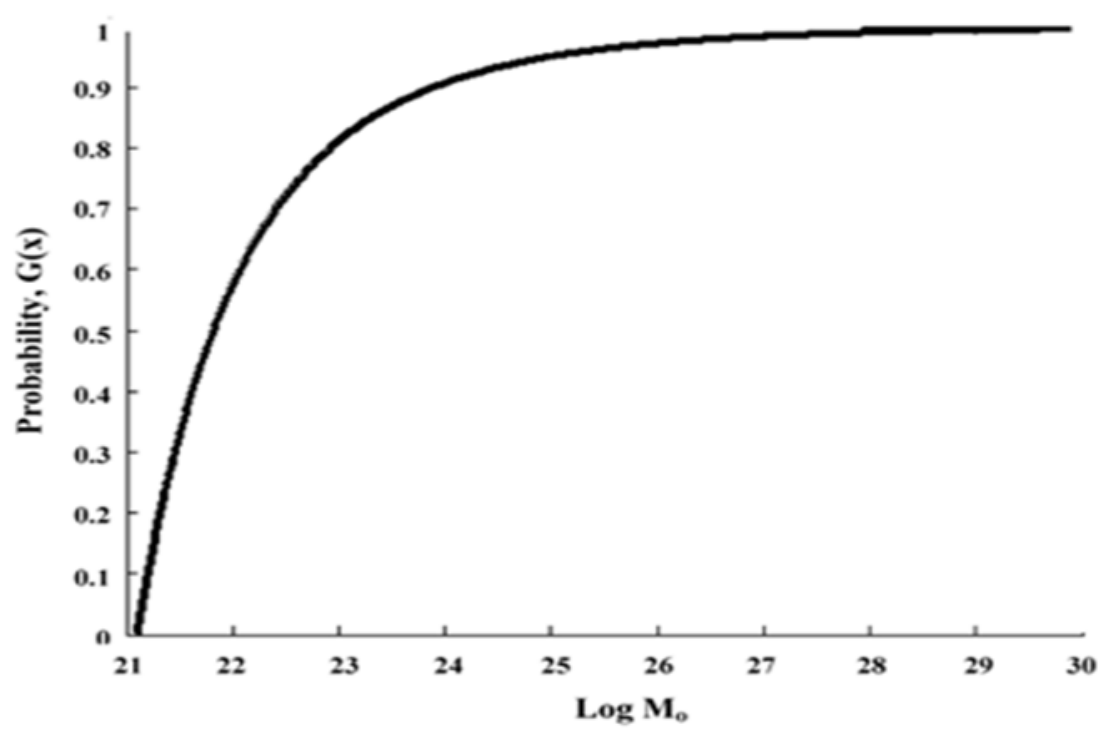

Fig. 7. Distribution function $G(x)=1-(5.1 /(x-16))^{5.25}$ for $x \geq 21.1$.

$$
H(y)=P\left(M_{0} \leq y\right)=P\left(\log M_{0} \leq \log y\right) .
$$

However, $Y=\log M_{0}$, and consequently

$$
H(y)=P(Y \leq \log y)=G(\log y) .
$$

Therefore

$$
H(y)= \begin{cases}1-\left(\frac{5.1}{\log y-16}\right)^{5.25} & \text { if } \quad \log y \geq 21.1 \quad\left(y \geq 10^{21.1}\right) \\ 0 & \text { else }\end{cases}
$$

Figure 8 represents the distribution function $Z=M_{0}$. To find the distribution functions of the random variables $Y_{\max }$ and $Z_{\max }$, we introduce the notation: $Y_{\max }=\max Y_{i}=\max \log M_{0 i}$ and $Z_{\max }=\max Z_{i}=\max M_{0 i}$ and denote by $G_{\max }(x)$ and $H_{\max }(y)$ their distribution functions, respectively, where

$$
Y_{\max }=\max \left(Y_{1}, Y_{2}, \ldots, Y_{n}\right)
$$

and

$$
Z_{\max }=\max \left(Z_{1}, Z_{2}, \ldots, Z_{n}\right) .
$$




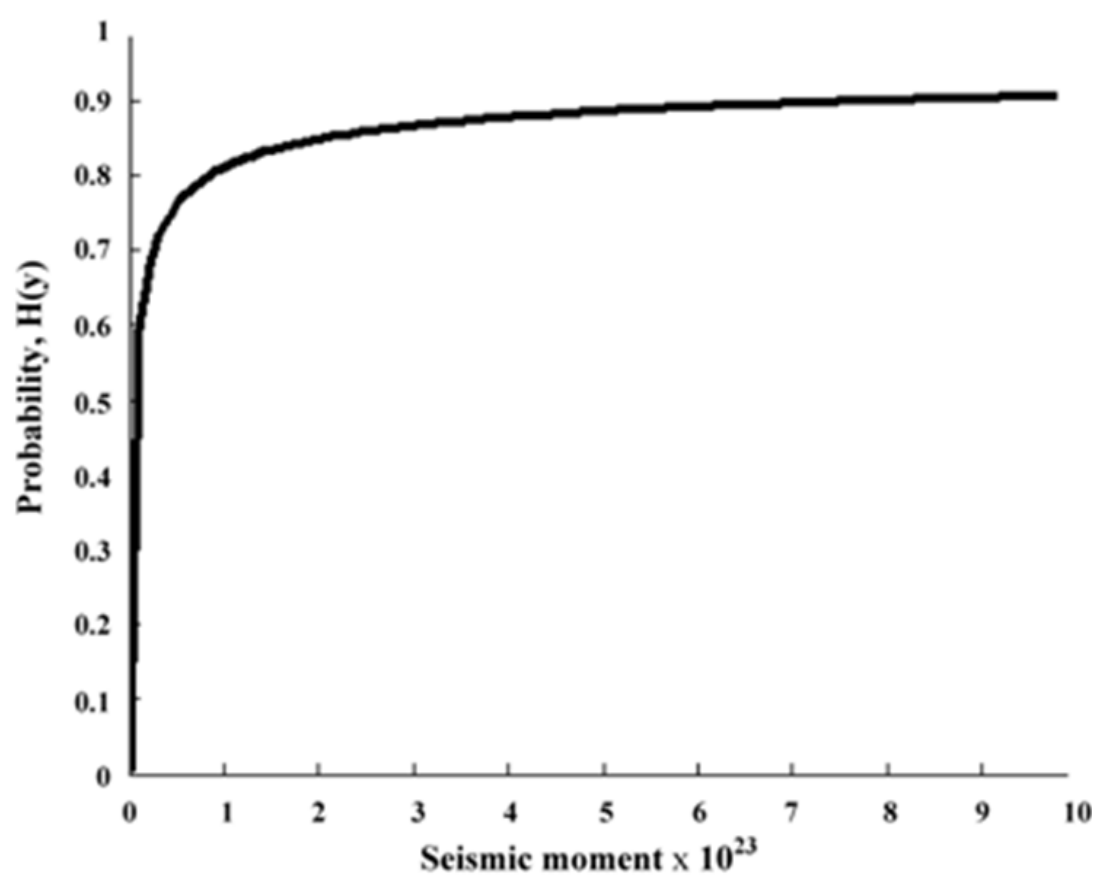

Fig. 8. Distribution function $H(y)=1-\left(5.1 /\left(\log _{10}(y)-16\right)\right)^{5.25}$ for $y \geq 10^{21.1}$.

We determined the quantiles of a given $p$ of $G(x), H(y)$ and the sufficient number of events $n$ so that the random variables $Y_{\max }$ and $Z_{\max }$ exceed a certain threshold with a given probability. In mathematical terms, we determine $x_{p}$ an $y_{p}$ solutions of the equations $G\left(x_{p}\right)=p$ and $H\left(y_{p}\right)=p$; then we calculate $n$ such as: $\quad P\left(Y_{\max } \geq x_{0}\right)=p \Leftrightarrow 1-P\left(Y_{\max } \leq x_{0}\right)=1-\left(G\left(x_{0}\right)\right)^{n} \quad$ and $P\left(Z_{\max } \geq m_{0}\right)=p \Leftrightarrow 1-P\left(Z_{\max } \leq m_{0}\right)=1-\left(H\left(m_{0}\right)\right)^{n}$. If $P\left(Y \leq x_{p}\right)=p$ and $P\left(Z \leq y_{p}\right)=p$, the quantiles $x_{p}$ and $y_{p}$ are given by

$$
\begin{aligned}
& x_{p}=16+5.1 \cdot q^{-\frac{1}{5.25}} \\
& y_{p}=10^{16} \cdot 10^{5.1 . q^{\frac{-1}{255}}}
\end{aligned}
$$

with $q=1-p$.

\section{PROBABILITIES OF EXPECTED EARTHQUAKES}

Table 6, computed by Eq. 14, gives a number $n$ of sufficient earthquakes which is enough to start, at $99.99 \%$ at least, the main seismic shock of a magnitude $M_{i} \geq m_{j}(j=\overline{1-50})$. Table 7 gives the number of earthquakes of 
Table 6

Number $n$ of earthquakes of magnitude $m \geq 3.4$ which is enough to cause an earthquake of a given magnitude and a given probability of $99.99 \%$

\begin{tabular}{|c|c|c|c|}
\hline$m_{j}$ & $n$ & $m_{j}$ & $n$ \\
\hline 3.5 & 4 & 6.3 & 230 \\
\hline 3.6 & 6 & 6.4 & 250 \\
\hline 3.7 & 9 & 6.5 & 272 \\
\hline 3.8 & 11 & 6.6 & 295 \\
\hline 3.9 & 14 & 6.7 & 319 \\
\hline 4.0 & 16 & 6.8 & 345 \\
\hline 4.1 & 19 & 6.9 & 373 \\
\hline 4.2 & 23 & 7.0 & 403 \\
\hline 4.3 & 26 & 7.1 & 435 \\
\hline 4.4 & 30 & 7.2 & 468 \\
\hline 4.5 & 35 & 7.3 & 504 \\
\hline 4.6 & 40 & 7.4 & 541 \\
\hline 4.7 & 45 & 7.5 & 581 \\
\hline 4.8 & 51 & 7.6 & 623 \\
\hline 4.9 & 58 & 7.7 & 668 \\
\hline 5.0 & 65 & 7.8 & 715 \\
\hline 5.1 & 72 & 7.9 & 765 \\
\hline 5.2 & 81 & 8.0 & 818 \\
\hline 5.3 & 90 & 8.1 & 873 \\
\hline 5.4 & 99 & 8.2 & 932 \\
\hline 5.5 & 109 & 8.3 & 993 \\
\hline 5.6 & 121 & 8.4 & 1058 \\
\hline 5.7 & 134 & 8.5 & 1126 \\
\hline 5.8 & 147 & 8.6 & 1198 \\
\hline 5.9 & 161 & 8.7 & 1273 \\
\hline 6.0 & 177 & 8.8 & 1352 \\
\hline 6.1 & 193 & 8.9 & 1435 \\
\hline 6.2 & 211 & 9.0 & 1522 \\
\hline
\end{tabular}

magnitude $m \geq 3.5$, which is enough to cause an earthquake of a given magnitude and a given probability; e.g., we determinate $n$ such as $p\left(M_{n} \geq m\right)=p$ with $M_{n}=\max _{i=1, n} M_{i}$, where $M_{i}$ is a random variable of the magnitude of the i-th earthquake, the value of $n$ being the intersection of the line $m$ and the column $p$. For the probability $p=0.9999$ and the magnitude $M_{0}=6.9$, we have found $n=373$. Thus, we can conclude that when earthquakes of magnitude $m \geq 3.4$ reach the number of 373 , it is possible with a probability of $99.99 \%$ to have an earthquake of a magnitude higher than 6.9 . For the prob- 
Table 7

Number $n$ of earthquakes of magnitude $m \geq 3.4$ which is enough to cause an earthquake of a given magnitude and a given probability

\begin{tabular}{|c|c|c|c|c|c|c|}
\hline $\begin{array}{c}p \\
\text { Magnitudes } \\
m\end{array}$ & $\begin{array}{c}p=0.9999 \\
(99.99 \%) \\
\text { Number } n\end{array}$ & $\begin{array}{c}p=0.9500 \\
(95 \%) \\
\text { Number } n\end{array}$ & $\mid \begin{array}{c}p=0.9000 \\
(90 \%) \\
\text { Number } n\end{array}$ & $\begin{array}{c}p=0.8500 \\
(85 \%) \\
\text { Number } n\end{array}$ & $\begin{array}{c}p=0.8000 \\
(80 \%) \\
\text { Number } n\end{array}$ & $\begin{array}{c}p=0.7500 \\
(75 \%) \\
\text { Number } n\end{array}$ \\
\hline 3.5 & 5 & 2 & 2 & 1 & 1 & 1 \\
\hline 3.6 & 7 & 3 & 2 & 2 & 2 & 2 \\
\hline 3.7 & 9 & 3 & 3 & 2 & 2 & 2 \\
\hline 3.8 & 12 & 4 & 3 & 3 & 2 & 2 \\
\hline 3.9 & 14 & 5 & 4 & 3 & 3 & 3 \\
\hline 4.0 & 17 & 6 & 5 & 4 & 3 & 3 \\
\hline 4.1 & 20 & 7 & 5 & 5 & 4 & 3 \\
\hline 4.2 & 24 & 8 & 6 & 5 & 5 & 4 \\
\hline 4.3 & 27 & 9 & 7 & 6 & 5 & 5 \\
\hline 4.4 & 31 & 11 & 8 & 7 & 6 & 5 \\
\hline 4.5 & 36 & 12 & 9 & 8 & 7 & 6 \\
\hline 4.6 & 41 & 14 & 11 & 9 & 8 & 7 \\
\hline 4.7 & 46 & 15 & 12 & 10 & 8 & 7 \\
\hline 4.8 & 52 & 17 & 13 & 11 & 10 & 8 \\
\hline 4.9 & 59 & 19 & 15 & 12 & 11 & 9 \\
\hline 5.0 & 65 & 22 & 17 & 14 & 12 & 10 \\
\hline 5.1 & 73 & 24 & 19 & 15 & 13 & 11 \\
\hline 5.2 & 82 & 27 & 21 & 17 & 15 & 13 \\
\hline 5.3 & 91 & 30 & 23 & 19 & 16 & 14 \\
\hline 5.4 & 100 & 33 & 25 & 21 & 18 & 16 \\
\hline 5.5 & 111 & 36 & 28 & 23 & 20 & 17 \\
\hline 5.6 & 122 & 40 & 31 & 26 & 22 & 19 \\
\hline 5.7 & 135 & 44 & 34 & 28 & 24 & 21 \\
\hline 5.8 & 148 & 48 & 37 & 31 & 25 & 23 \\
\hline 5.9 & 162 & 53 & 41 & 34 & 29 & 25 \\
\hline 6.0 & 178 & 58 & 45 & 37 & 31 & 27 \\
\hline 6.1 & 194 & 63 & 49 & 40 & 34 & 30 \\
\hline 6.2 & 213 & 69 & 53 & 44 & 37 & 32 \\
\hline 6.3 & 231 & 75 & 58 & 48 & 41 & 35 \\
\hline 6.4 & 251 & 82 & 63 & 52 & 44 & 38 \\
\hline 6.5 & 273 & 89 & 58 & 57 & 48 & 41 \\
\hline 6.6 & 296 & 96 & 74 & 61 & 52 & 45 \\
\hline 6.7 & 320 & 104 & 80 & 66 & 56 & 49 \\
\hline
\end{tabular}


Table 7 (continuation)

\begin{tabular}{|c|c|c|c|c|c|c|}
\hline Magnitudes & $\begin{array}{c}p=0.9999 \\
(99.99 \%) \\
\text { Number } n\end{array}$ & $\begin{array}{c}p=0.9500 \\
(95 \%) \\
\text { Number } n\end{array}$ & $\mid \begin{array}{c}p=0.9000 \\
(90 \%) \\
\text { Number } n\end{array}$ & $\begin{array}{c}p=0.8500 \\
(85 \%) \\
\text { Number } n\end{array}$ & $\begin{array}{c}p=0.8000 \\
(80 \%) \\
\text { Number } n\end{array}$ & $\begin{array}{c}p=0.7500 \\
(75 \%) \\
\text { Number } n\end{array}$ \\
\hline 6.8 & 346 & 113 & 87 & 72 & 61 & 53 \\
\hline 6.9 & 374 & 122 & 94 & 77 & 66 & 57 \\
\hline 7.0 & 404 & 132 & 101 & 84 & 71 & 61 \\
\hline 7.1 & 436 & 141 & 109 & 90 & 77 & 66 \\
\hline 7.2 & 469 & 153 & 118 & 97 & 82 & 71 \\
\hline 7.3 & 505 & 164 & 127 & 104 & 89 & 76 \\
\hline 7.4 & 542 & 177 & 136 & 116 & 95 & 82 \\
\hline 7.5 & 582 & 190 & 146 & 120 & 102 & 88 \\
\hline 7.6 & 624 & 203 & 156 & 129 & 110 & 94 \\
\hline 7.7 & 669 & 218 & 168 & 138 & 117 & 101 \\
\hline 7.8 & 716 & 233 & 179 & 148 & 126 & 108 \\
\hline 7.9 & 766 & 249 & 192 & 158 & 134 & 116 \\
\hline 8.0 & 819 & 267 & 205 & 169 & 143 & 124 \\
\hline 8.1 & 874 & 285 & 219 & 180 & 153 & 132 \\
\hline 8.2 & 932 & 304 & 233 & 192 & 163 & 141 \\
\hline 8.3 & 994 & 324 & 249 & 205 & 174 & 150 \\
\hline 8.4 & 1059 & 345 & 265 & 218 & 185 & 160 \\
\hline 8.5 & 1127 & 367 & 282 & 233 & 197 & 170 \\
\hline 8.6 & 1199 & 390 & 300 & 247 & 210 & 181 \\
\hline 8.7 & 1274 & 415 & 319 & 263 & 223 & 192 \\
\hline 8.8 & 1353 & 440 & 339 & 279 & 237 & 204 \\
\hline 8.9 & 1436 & 467 & 359 & 296 & 251 & 217 \\
\hline 9.0 & 1523 & 496 & 381 & 314 & 266 & 230 \\
\hline
\end{tabular}

ability $p=0.9999$ and the magnitude $M_{0}=8$, we have found $n=818$ and we can also conclude that when earthquakes of magnitude $m \geq 3.4$ reach the number of 818 , we are at a probability of $99.99 \%$ possible to expect an earthquake of a magnitude higher than 8 . We can notice that the two formulas, 10 and 11, give the same probabilities. For the determination of $n=373$ and $n=818$, it is enough to substitute $p$ by $0.9999, M_{0}=6.9$ and $M_{0}=8$, respectively, in formula 14. For the probability given by the Fréchet law, we can substitute $n$ by 373 and 818 , as well as $M_{0}$ by 6.9 and by 8 in relation 12 , and we can obtain $x=0.65693269$ and $x=0.655844556$, respectively. Then, we can substitute $m$ by these numbers in relation 11, and we obtain $p=0.99988591$ and $p=0.999894614$, respectively. The two results correspond almost to a probability of $99.99 \%$. 
Relations 21 and 24 permitted us to know the probability to forecast future earthquakes. Table 8 gives the number $n$ of sufficient earthquakes to affirm, with a given probability $p$, that it will be one event whose decimal logarithm of its moment exceeds a given threshold $x_{0 p}$. With the same principle, Table 9 gives the number of earthquakes so that the seismic moment variable $Z_{\max }$ exceeds a certain threshold for a given probability. We note that an event of some magnitude can occur before the number $n$ of earth-

Table 8

Distribution function $H(y)=1-\left(5.1 /\left(\log _{10}(y)-16\right)\right)^{5.25}$ for $y \geq 10^{21.1}$

\begin{tabular}{|c|c|c|c|c|c|c|c|c|c|c|}
\hline$p$ & $99.99 \%$ & $99.95 \%$ & $99.9 \%$ & $99.5 \%$ & $99 \%$ & $95 \%$ & $90 \%$ & $85 \%$ & $80 \%$ & $75 \%$ \\
\hline 22.0 & 17 & 14 & 13 & 10 & 9 & 6 & 5 & 4 & 3 & 3 \\
23.0 & 44 & 37 & 33 & 26 & 22 & 15 & 11 & 10 & 8 & 7 \\
24.0 & 94 & 77 & 70 & 54 & 47 & 31 & 24 & 20 & 17 & 15 \\
25.0 & 178 & 147 & 133 & 102 & 89 & 58 & 45 & 37 & 31 & 27 \\
26.0 & 312 & 257 & 234 & 180 & 156 & 102 & 78 & 65 & 55 & 47 \\
26.5 & 404 & 333 & 303 & 233 & 202 & 132 & 101 & 84 & 71 & 61 \\
26.75 & 458 & 378 & 343 & 263 & 229 & 149 & 115 & 95 & 80 & 69 \\
27.0 & 517 & 427 & 388 & 298 & 259 & 168 & 130 & 107 & 91 & 78 \\
27.25 & 582 & 480 & 437 & 335 & 291 & 190 & 146 & 120 & 102 & 88 \\
27.5 & 654 & 540 & 491 & 376 & 327 & 213 & 164 & 135 & 115 & 99 \\
27.75 & 732 & 605 & 549 & 422 & 366 & 239 & 183 & 151 & 128 & 111 \\
\hline
\end{tabular}

Table 9

Determination of the number $n$ such as $P\left(Z_{\max } \geq m_{0}\right)=p$

\begin{tabular}{|c|c|c|c|c|c|c|c|c|c|c|}
\hline $\begin{array}{c}m_{0} \\
\text { [dyne cml }\end{array}$ & $99.99 \%$ & $99.95 \%$ & $99.9 \%$ & $99.5 \%$ & $99 \%$ & $95 \%$ & $90 \%$ & $85 \%$ & $80 \%$ & $75 \%$ \\
\hline $10^{22}$ & 17 & 14 & 13 & 10 & 9 & 6 & 5 & 4 & 3 & 3 \\
$10^{23}$ & 44 & 37 & 33 & 26 & 22 & 15 & 11 & 10 & 8 & 7 \\
$10^{24}$ & 94 & 77 & 70 & 54 & 47 & 31 & 24 & 20 & 17 & 15 \\
$10^{25}$ & 178 & 147 & 133 & 102 & 89 & 58 & 45 & 37 & 31 & 27 \\
$10^{26}$ & 312 & 257 & 234 & 180 & 156 & 102 & 78 & 65 & 55 & 47 \\
$3.16 .10^{26}$ & 404 & 333 & 303 & 233 & 202 & 132 & 101 & 84 & 71 & 61 \\
$5.62 .10^{26}$ & 458 & 378 & 343 & 263 & 229 & 149 & 115 & 95 & 80 & 69 \\
$10^{27}$ & 517 & 427 & 388 & 298 & 259 & 168 & 130 & 107 & 91 & 78 \\
$1.78 .10^{27}$ & 582 & 480 & 437 & 335 & 291 & 190 & 146 & 120 & 102 & 88 \\
$3.16 .10^{27}$ & 654 & 540 & 491 & 376 & 327 & 213 & 164 & 135 & 115 & 99 \\
$5.62 .10^{27}$ & 732 & 605 & 549 & 422 & 366 & 239 & 183 & 151 & 128 & 111 \\
\hline
\end{tabular}


quakes associated with it is reached, but with a lower probability. It is enough to observe the tables to realize that the number of earthquakes decreases as the decay of the fixed probabilities. The time interval between strong earthquakes or the seismic cycle is expected to increase along with the magnitude increase as well. The seismic cycle depends on the earthquake energy or magnitude, which, in first approximation, is proportional to a crust block volume that has accumulated a certain potential of elastic energy. The latter result does not contradict the data obtained experimentally (Baddari and Frolov 2010, Sobolev and Ponomarev 2003). This implies that a stronger earthquake takes more time to be generated and is forecasted by weak seismicity.

\section{STATISTICAL CHARACTERISTICS OF THE SEISMIC REGIME}

\subsection{Recurrence plot slop and the fractal dimension}

A tectonic earthquake is a multi stage process. It may be considered as a final step in a determined phase of a discrete failure stochastic process in Earth crust. This process leads to total or partial stress release over the fracture area and continues to be developed in different stresses field. We used the seismic recursive general law with fractal property of seismicity as (Baddari and Frolov 2010, Smirnov et al. 1995)

$$
\log N=-\gamma \log E_{S}+D \log Y+D_{t} \log t+\tau
$$

where

$$
\begin{aligned}
& \tau=\gamma \log a-D \log \rho-D_{t} \log \psi, \\
& \gamma=\frac{c D+\zeta D_{t}}{\alpha}
\end{aligned}
$$

$E_{s}$ is the seismic energy $E_{s}=a L_{i}^{\alpha}$, defined by its source dimension $L_{i}$ with $\alpha \approx 3$ in average (Kasahara 1985, Sadovsky et al. 1991), $Y$ is the size of the seismoactive region, $\rho, c, \zeta$, and $\psi$ are certain constants, $t$ is the seismic cycle. The deduced relation between the recurrence plot slope $\gamma$, the fractal dimension $D$ and the temporal fractal dimension $D_{t}$ is:

$$
\alpha \gamma-c D-\zeta D_{t}=0
$$

Supposing $\alpha \approx 3$ and fixing $c=1$ (Smirnov et al. 1995, Baddari and Frolov 1997), we get

$$
D=3 \gamma-\zeta D_{t} .
$$


Experimentally, $\gamma \approx 0.5$ and $D \approx 1.5$ in average (Smirnov et al. 1995, Baddari and Frolov 2010); therefore, we can deduce that in Eq. 30 the constant $\zeta \approx 0$, since $D_{t}>0$ ( $D_{t}=0$ means that all earthquakes will occur simultaneously). The equality 30 is equivalent to $D=2 \gamma_{M}$, where $\gamma_{M}$ is the slope of the recurrence curve for magnitudes $\left(\gamma_{M}=1.5 \gamma\right)$ (Main et al. 1990). The recurrence plot slope $\gamma$ is calculated by the method of maximum likelihood. The statistical error of the $\gamma$-value was calculated as a square root of the asymptotic variance (Kendall and Stuart 1961, Smirnov et al. 2013): $S_{\gamma}=\gamma /(N)^{1 / 2}$, where $N$ is the number of seismic events used for estimation of $\gamma$. For the measure of the fractal dimension $D$ of a set of seismic events, we have used the cellular evaluation and the correlation and we have interpreted the correlation dimension as the fractal dimension, supposing that seismicity is a near-homogeneous fractal. The correlation dimension $D$ was calculated by applying the algorithm (Sobolev 1995, Baddari and Frolov 1997)

$$
D=\lim _{l \rightarrow 0} \frac{\log C(l)}{\log l}
$$

where $C(l)$ is the correlation integral defined as

$$
C(l)=N\left|\bar{r}_{i}-\bar{r}_{j}\right| \leq \frac{l}{m(m-1)}
$$

$N$ is the number of pairs of seismic events with distances between hypocenters less than $l$, and $m$ is the number of events. The statistical error of the correlation dimension was estimated by the direct estimation method known as jackknife technique and based on the influence function approach (Huber and Ronchetti 2011, Smirnov et al. 2013).

The estimates of the $\gamma$-value and the fractal dimension $D$ of the set of seismic events have been realized in a moving time window over the studied area. We used the windows covered 25 events and were shifted by 10 events. Small errors are noted in windowing process not exceeding \pm 0.05 . Changes have been recorded for the recurrence plot slope $\gamma$ in the studied seismically active area of Zemmouri seismoactive region. The $\gamma$-value decreased from 0.68 to 0.42 eighteen months before the seismic event of Zemmouri $(M=6.9)$ of 21 May 2003 (Fig. 9). The decrease is significant at a level of 0.26 . We noted a much smaller variability from the average value $(0.68$ \pm 0.05 ) in the recurrence graph slope during the period from 1980 up to December 2001. In this light, the $\gamma$-value variability seems to be most promising in med-term prognostication. The earthquake of Zemmouri revealed a diminution of the fractal dimension $D$ from 2.1 to 1.25 eighteen months before the principal event (Fig. 9). We did not notice any remarkable trend in 


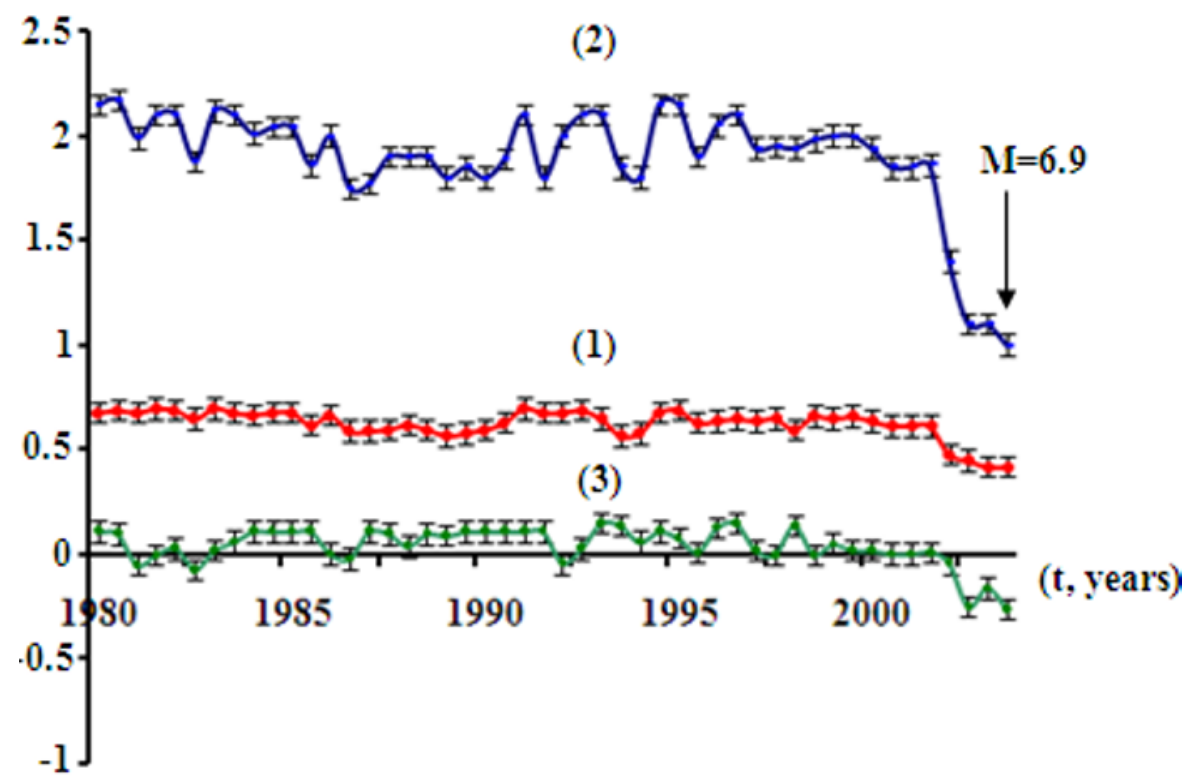

Fig. 9. Dependences of seismic regime parameters $\gamma(1), D(2)$, and $D-3 \gamma$ (3) on normalized time prior to the Zemmouri earthquake $M=6.9 . t_{s}$ corresponds to 23 years of the experimental field time.

the variation of the graph of the fractal dimension $D$ before December 2001 . Its average value was $2 \pm 0.4$ during the period from 1980 up to December 2001. $D$ decreased sharply to reach 1.1 many days before the main event. The decrease of $D$ can be explained by the progressive appearance of local instability in the deformed volume of materials as it approaches macrocracks. The decreases in $\gamma$ and $D$ values point to the redistribution of failure from the smaller scales to larger ones, which is typical of the preparation process of a tectonic earthquake (Scholz 2010, Sobolev 2011).

\subsection{Variations of the crack concentration parameter in the seismoactive regions}

Results of experimental investigations of failure show spatial distributions of cracks in rocks obey the fractal statistics (Sobolev 1995, Zavyalov 2006, Baddari et al. 2012, 2015). In a chaotic distribution of microcracks, the probability of the formation of a set of $x$ adjacent cracks, i.e., the transition from delocalization to localization, is

$$
p_{n}=\frac{\langle x\rangle^{x}}{x !}
$$


with the mean number $\langle x\rangle$ of cracks by cluster equal to $\langle x\rangle=x / K_{s r}$ and the mean inter-crack distance $K$ during their volumetric concentration per unit of size $N=x V^{-1}$ equal to

$$
K=N^{-1 / 3} / \bar{l},
$$

where $V$ is the volume of the confined rock and $\bar{l}$ is the mean size of the accumulated cracks given by $\bar{l}=\frac{1}{x} \sum_{i=1}^{x} l_{i}$, with $l_{i}$ the size of the crack. The exact value of the calescence criterion is $K^{*}=3.8$, which constitutes the threshold of the transition from the stage of crack accumulation to their start to coalesce and the formation ensembles of clusters in the cracked body (Baddari et al. 2015). We will use $K_{s r}$ instead of $K$ to characterize the crack concentration parameter of the seismogenic region. $K_{s r}$ decreased to 4.1 ten months before the principal seismic event of Zemmouri (Fig. 10). The mean value of $K_{s r}$ was $16 \pm 2$ up to August 2002. Changes in $K_{s r}$ during this period were not significant, which indicates that the accumulation of relatively small cracks (weak earthquakes) occurred in the studied territory. The sharp drop of $K_{s r}$ was clearly observed from September 2002, which testifies to an evolution of avalanche-like coalescence and enlargement of the cracks giving the occurrence of the main event of 21 May 2003. $K_{s r}$ reached 4.2 before

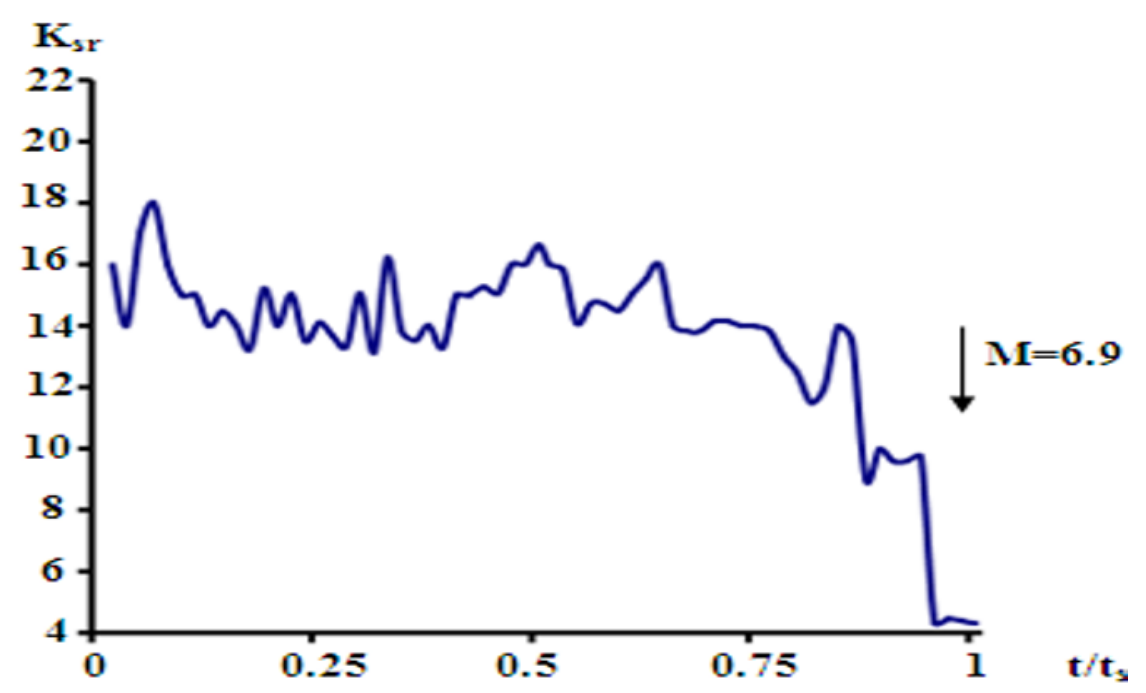

Fig. 10. Dependence of the parameter $K_{s r}$ on normalized time in $200 \times 200 \mathrm{~km}$ zone that contains the epicenter of $M=6.9$ of Zemmouri earthquake. $t_{s}$ corresponds to 23 years of the experimental field time. 
the earthquake of Kamchatka of 5 December $1997(M=7.9)$ (Zavyalov 2006). In other terms, when the parameter $K_{s r}$ attained the critical value of 4-7, the cracks clusterisation acquired a threshold character that caused a relatively quick fault expansion. A quasi-invariance of $K_{s r}$ is noted for zones which are far from epicenters, which confirms the hypothesis of spatial localization of SE in the level of the rupture zone. The values of $K_{s}$, before the macrorupture, calculated for the geophysical medium at different scales, are situated in the interval 3-7 (Baddari et al. 2015, Zavyalov 2006).

\subsection{Changes in percentage of clustering seismic events}

The analysis of the variation of variance coefficient of the number of seismic events during the waiting period of a powerful shock $(M=6.9)$ (Fig. 11) demonstrates that the gradual rise of ruptures in the epicentral zone is accompanied by an increase of the average magnitude $\bar{M}$ of premonitory seismic events $\left(M_{\min } \geq 2\right)$ preceding the main shock in the Zemmouri earthquake zone (Fig. 12). $\bar{M}$ varied from 2.35 till 2.43 during the period up to December 1998, increased till 2.48 in December 2000 and started to decrease down to 2.35 four to five weeks before the main earthquake. The above account testifies to the assumption that fractures tend to enlarge as the moment of major strong earthquake approaches.

We assumed that two successive seismic events are clustered if the critical distance $d_{c r}$ and the critical time $t_{c r}$ between them is less than the critical

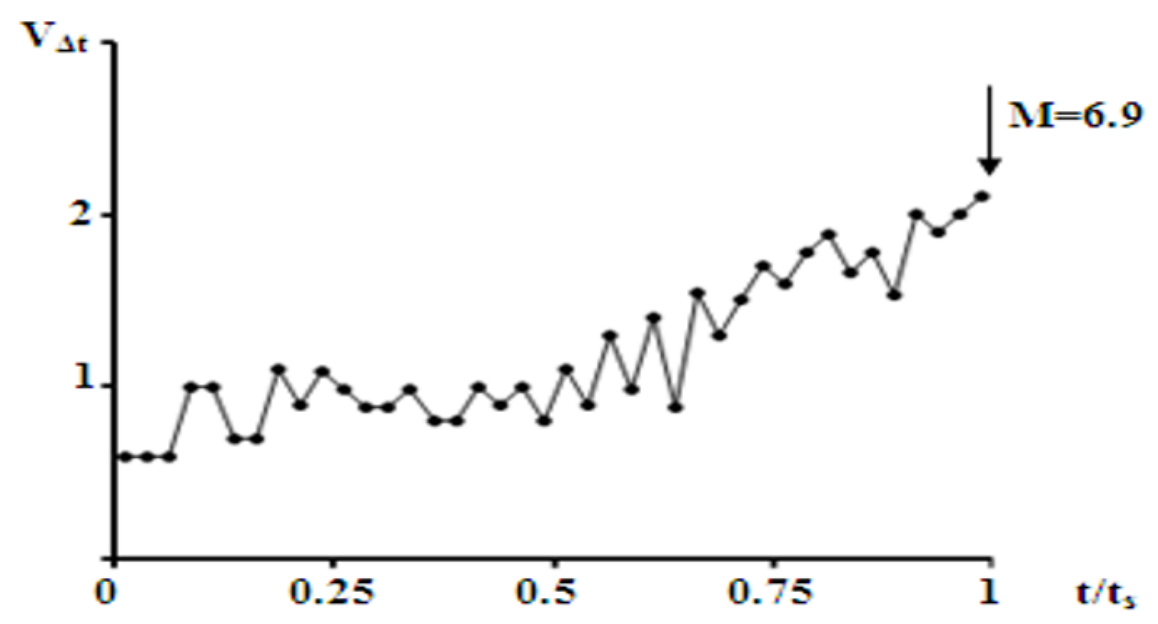

Fig. 11. Variation of variance coefficient of the number of small seismic events during the expectation period of Zemmouri earthquake. $t_{s}$ corresponds to the experimental field time (23 years). 


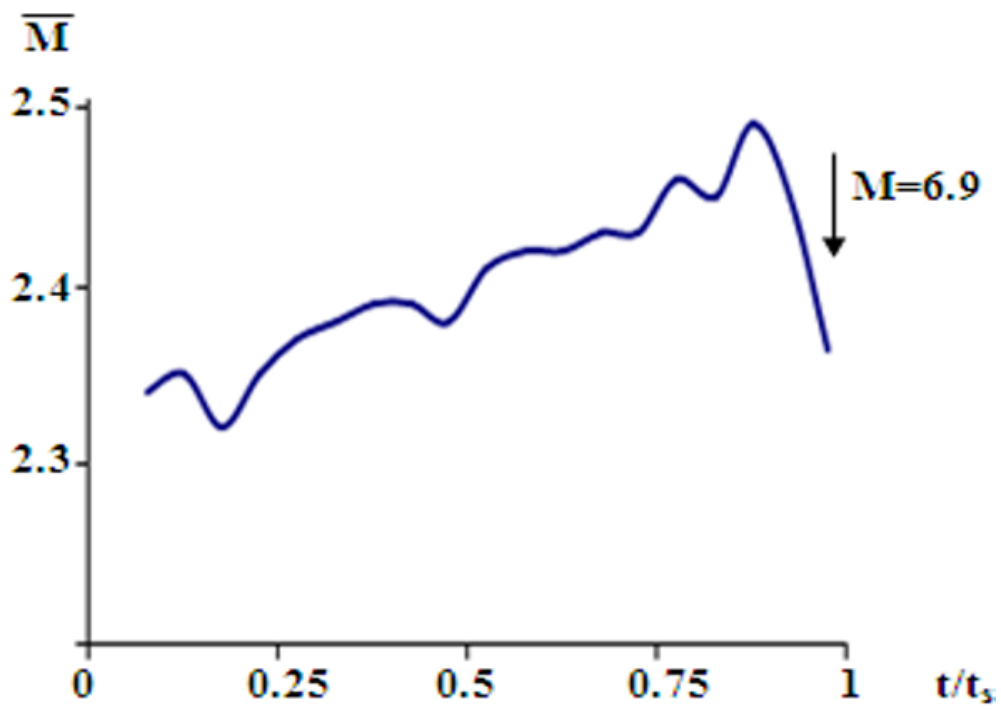

Fig. 12. Variation of the average magnitude $\bar{M}$ in Zemmouri area earthquake. $t_{s}$ corresponds to the experimental field time (23 years).

values corresponding to these two parameters (Baddari et al. 1996, Baddari and Frolov 2010, Sobolev 1995): $t_{c r}$ [hours $]=a 10^{b M}$ and $d_{c r}[\mathrm{~km}]=c L_{i}$, where $a=5.210^{4}, b=0.75$, and $c=3 ; M$ is the magnitude of the first earthquake in a pair of seismic events and $L_{i}$ is the length of a rupture in the epicenter of an earthquake of a given energy calculated by the empirical relation (Riznichenko 1976, Sobolev and Ponomarev 2003, Baddari and Frolov 2010): $\log L_{i}[\mathrm{~km}]=0.244 \log E_{s}(j)-2.266$, where $E_{s}$ is the energy of the earthquake. There were noticed three stages of the deformation of the Zemmouri geophysical medium in the variation of the clustering coefficient $G \%$ (Fig. 13). $G \%$ is the ratio of the number of clustering small earthquakes $N g\left(M_{\min } \geq 2\right)$ to their total number $\Sigma N$ in the studied seismogenic zone: $G \%=N g / \Sigma N$. As a result, three regularities of earthquake clusterization were revealed. The increase of $G \%$ in the stage $A$, reaching $12 \%$ ten years before the major event, is associated with the increase of interaction between single fractures in the epicentral area. The reduction in amount of $G \%$ during the stage $B$, reaching about $1 \%$ five years prior to a major event, is due to the strain and stress relaxation. The rapid growth of $G \%$ during the stage $C$, reaching $13-15 \%$ days or weeks before a major event, is from clustering of single fractures and formation of the seismic main nucleus of the 2003 Zemmouri earthquake. This may be due to failure of relatively strong barriers that prevented a dynamic spread of the fault which is responsible of the coming strong earthquake. 


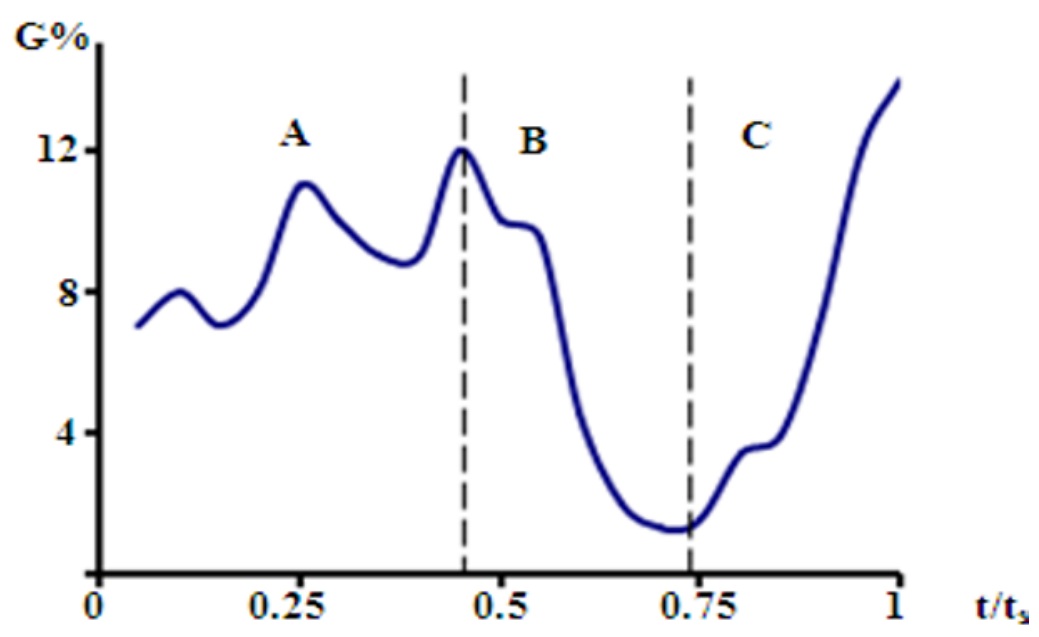

Fig. 13. Changes in percentage of grouping earthquakes $G \%$ in the epicentral area of Zemmouri earthquake.

\subsection{The Hurst exponent}

Generally, the Hurst component $H$ can be any real number between 0 and 1 (Hurst 1965, Smirnov et al. 1995). For $H>0.5$, the temporal series reveals persistence phenomenon, which increases as $H$ approximates 1 . For $H<0.5$, a series shows an anti-persistence phenomenon. Drift of $H$ from 0.5 value may serve as a precursor of temporal connectivity of the state of the physical system triggering the process. The computation of $H$ exponent was made for two cases: $H=H_{r}$, i.e., a distance between hypocenters of two consecutive events when the temporal series $z(t)=\left|r\left(t_{i}\right)-r\left(t_{i-1}\right)\right|$, and for $H=H_{t}$, i.e., a time interval between two consecutive events when $z(t)=t_{i}-t_{i-1}$. The Hurst exponents were calculated in a window which included $T$ points and was covered by nonintersecting small windows $\tau_{k}(k=1,2, \ldots)$. Figures 14-15 show that $H_{t}$ and $H_{r}$ started to increase many months before the principal seismic event of Zemmouri epicentral zone. $H_{r}$ and $H_{t}$ reached 0.68 and 0.65 , respectively, 2-3 weeks before the main shock of 21 May $2003(M=6.9) . H_{r}$ and $H_{t}$ maintained their values of 0.5 during 17 years till December 1998 and started to mildly increase up to the main shock of May 2003. Significant increments of $H_{r}$ and $H_{t}$, reaching 0.6, were observed 33-35 months preceding the strong earthquake. In other terms, when the parameters $H_{t}>0.5$ and $H_{r}>0.5$, the fractures clusterization acquired a certain structure that caused a relatively quick fault expansion. The deformation of the geophysical field was related to the continuation of accumulation of energy which differs from an active zone to another seismic zone by the speed of motion of block rocks and dissipation of energy at different hierarchy levels of the medium. 


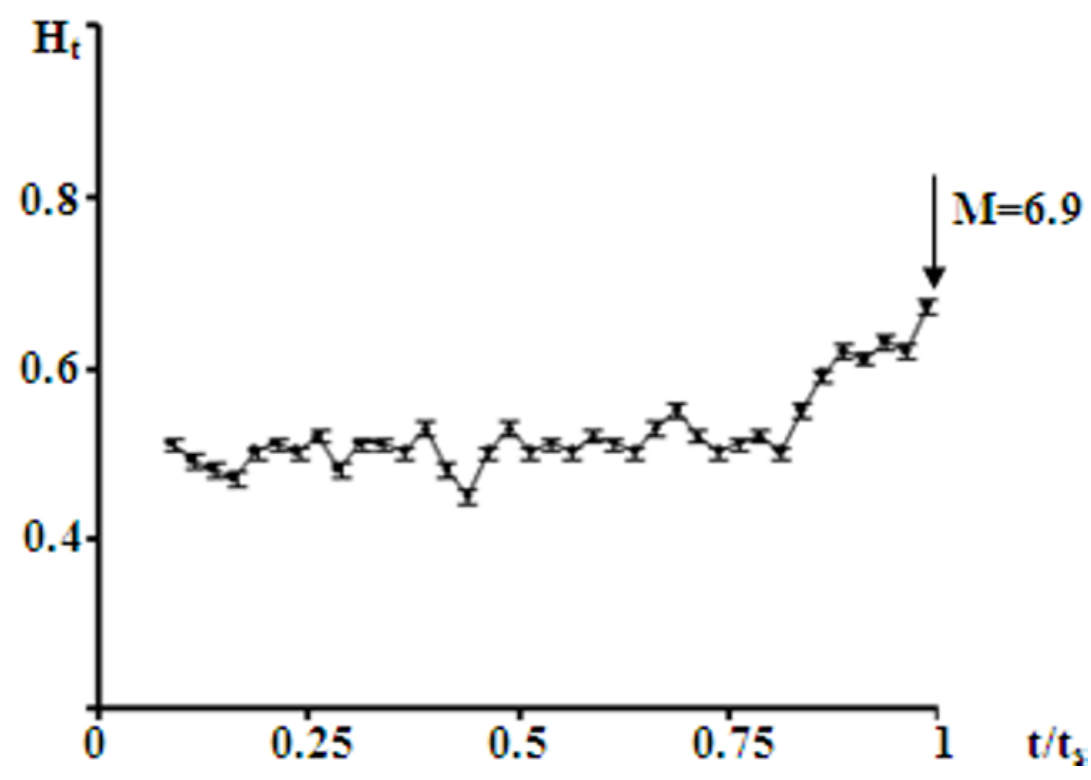

Fig. 14. Changes in Hurst exponent $H_{t}$ during the expectation period in the epicentral area of Zemmouri earthquake.

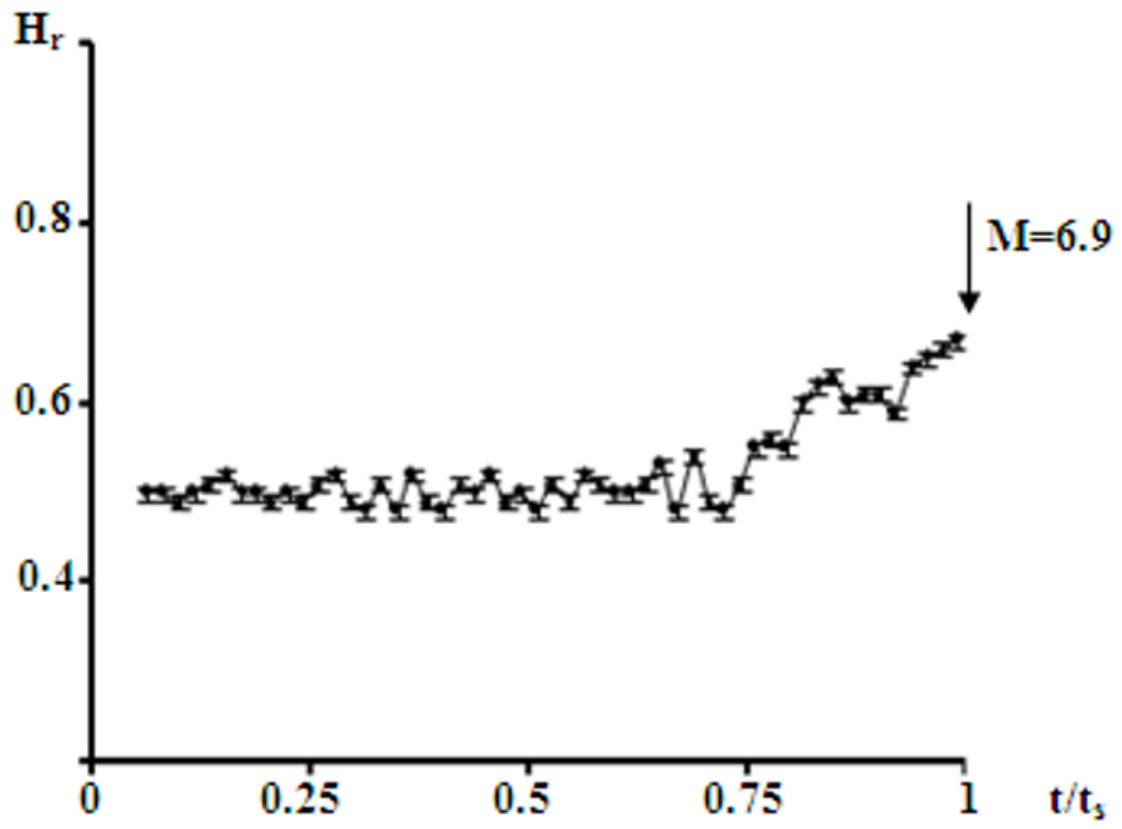

Fig. 15. Changes in Hurst exponent $H_{r}$ during the expectation period in the epicentral area of Zemmouri earthquake. 


\section{DISCUSSION}

The magnitudes follow the Pareto law. The magnitude distribution function has been determined using the $\chi^{2}$ and KS adjustment tests. These nonparametric tests are less constraining, particularly concerning the studied sample size. According to the Von Mises criterion, the Pareto distribution function belongs to the attraction field of the Fréchet limiting law. It should be remembered that the Pareto distribution has been used by numerous researchers, in particular for studies of the extreme value laws (Csikor et al. 2007, Dahmen et al. 2009). The developed methods give the probability of an earthquake of a maximum energy as the result of coalescence of several smaller seismic shocks. We note that an event of some magnitude can occur before reaching the number $n$ of earthquakes associated with it, but with a lower probability.

It should be noted that some constraints affect the magnitude scales in the case of a large local or regional seismic event, such as saturation and discrepancies between scales. The moment magnitude scale is physically transparent, and it is reliable to predict source parameters and strong motion amplitudes (Gusev 1991, Utsu 2002). For this reason, the moment magnitude has been used in our model. The statistical estimates of the failure cycle of Zemmouri region are based on the Pareto distribution, which depends on the number of the events with a given magnitude moment threshold (small or low earthquakes). Given the correlation existing between the seismic moment and the magnitudes, probability laws of the distribution functions of the seismic moment were deduced. Some forecast moments of the future earthquakes in the studied area were given. The proposed method makes it possible to determine the required time for which an earthquake may occur with seismic moment greater than a threshold $m_{o}$ with a given probability $p$. The maximum expected value of the magnitude depends on the considered geological structure of the field and the seismotectonic processes proceeding in the Earth's crust in the Algerian seismoactive area. The irregular distribution of seismic focuses in the area of Zemmouri is related to the discrete structure of the geophysical field. This discreteness remains unchanged over long intervals of time. The return cycles of the large earthquakes in this area under study depend on several conditions existing in the geophysical system in the Earth crust.

The processes of preparation of the earthquake source are reflected in the variation of statistical features of the seismic regime, which can be referred as prognostic anomalies. The decreases of $\gamma, D$, and $K_{s r}$ values are caused by coalescing cracks and the formation of larger ones. The decreases are of about $20-80 \%$ with respect to their habitual values 10 to 18 months before the principal seismic shock. The diminution of $\mathrm{D}$ advanced the decrease of $\gamma$. 
The values of $H_{t}$ and $H_{r}$ increase, reaching 0.6, 33-35 months before the strong earthquake. This increments of $H_{t}$ and $H_{r}\left(H_{t}>0.5\right.$ and $\left.H_{r}>0.5\right)$ are indicative of persistent behavior of seismicity. Similar results were obtained by Zavyalov (2006), Console et al. (2007), Scholz, (2010), Smirnov et al. (2013). The parameters $D, \gamma, K_{s r}, H_{r}$, and $H_{t}$ of geometric set of seismic events reflect the variation of seismic regime. The antiphased variations of $D, \gamma, K_{s r}$, and $H_{r}, H_{t}$ are associated to the gradual clusterization and the expansion of the fractal structure in the geophysical medium under stress.

In other words, the number of the weakest events diminished due to the reduction of the small scale rupture in the rock masses, i.e., the transition of fracturing from lower to higher levels. This conclusion agrees with results of similar analysis obtained by Sobolev (2011), and Potanina et al. (2015). The interruption of the equivalence $D \approx 3 \gamma$ may be related to the redistribution of medium stress-strain state as a function of the rupture dimension and of seismic regime evolution preceding and succeeding the principal shock. We note a gradual reinforcement of the ruptures in the formation failure zone accompanied by the increase of the mean magnitude $\bar{M}$ of week seismic events preceding the principal shock in the zone of Zemmouri. Three stages of the deformation of the geophysical medium are manifested in the variation of the coefficient $G \%$ of the clustering of minor seismic events. The mass of cluster means the number of seismic events that fill the area of the earthquake preparation zone. This allows us to say that in each seismic part having a rank situated at a fixed set of seismic fractals of lower rank recur independently of this part's dimension.

\section{CONCLUSION}

a The $\chi^{2}$ and KS adjustment tests have been used to adjust the Zemmouri seismic data using a distribution function. The magnitudes follow the Pareto law. The results showed that the used Pareto distribution function belongs to the attraction field of the Fréchet law. The limiting extreme values law confirmed the correctness of the adjustment method. The probabilities of various expected earthquakes were computed. Using the moment magnitude scale, a probabilistic model was made to predict the occurrences of strong earthquakes.

- The $\gamma$ and $D$ values remain constant, and then decrease until the triggering of the macrofailure (earthquake). The equivalency $D \approx 3 \gamma$ appeared in the first stage of the seismic cycle, when the stress fields of dispersed seismic events did not overlap and interact. This equivalency started to be violated 18 months before the major earthquake. Lower values of crack concentration parameter $K_{s r}$ were observed 10 months before the main seismic events. $H_{t}$ and $H_{r}$ exponents started to increase 33-35 months be- 
fore the main shock. The clusterization parameter $G \%$ showed three stages in the seismic regime evolution: rise, decrease, and renewed rise prior to the main shock. The average magnitude $\bar{M}$ of microearhquakes was raised up to the moment of powerful shock. In this light, the significant increments of the studied statistical features indicate that seismic events are clustering in space and seismic states become connected in time many months before the expected strong dynamic event, which corresponds to the stage of crack fusion and growth.

Acknowledgments. The authors would like to express their gratitude to the two anonymous referees for their fruitful and valuable comments.

\section{Appendix}

\section{Other descriptive statistics}

Figure A1 shows the cumulative number of earthquakes with magnitude $M s \geq 2.0$ versus time. Linear trends of seismicity can be depicted for the following time intervals: 1981-1990 and 1990-2001. Starting from 2001, the number of earthquakes has been increasing abruptly as a consequence of improvements of Algerian seismic network.

In Fig. A2, we present a histogram of the number of earthquakes per year. The figure shows that most $(73 \%)$ of the events occurred in the second half of the observation period. Figure A3 depicts the variation of magnitude of completeness with time. The magnitude of completeness is calculated using the maximum curvature method of Woessner and Wiemer (2005); we applied a running window technique with 50 events per window and a step of 10 events.

We can observe that during the interval 1985-2001 (Fig. A3) Mc mostly oscilated between 3.0 and 3.4. Starting from the year 2001, $M$ has surprisingly low values close to 2.4 . This low threshold is almost certainly a result of improving seismic-network performance (e.g., increasing number of stations), leading to greater magnitude sensitivity.

Figure A4 depicts the frequency-magnitude relation for the studied source region. Based on maximum curvature procedure (MAXC), the magnitude of completeness was taken equal to 2.8. Using these threshold magnitudes, we derived the $\gamma$ value of the Gutenberg-Richter relationship and its standard deviation using the maximum likelihood procedure. To estimate the standard error in $\gamma$ we use the formulae given by Shi and Bolt (1982). Overall, we find $\gamma=0.68 \pm 0.05$ for our studied events. 


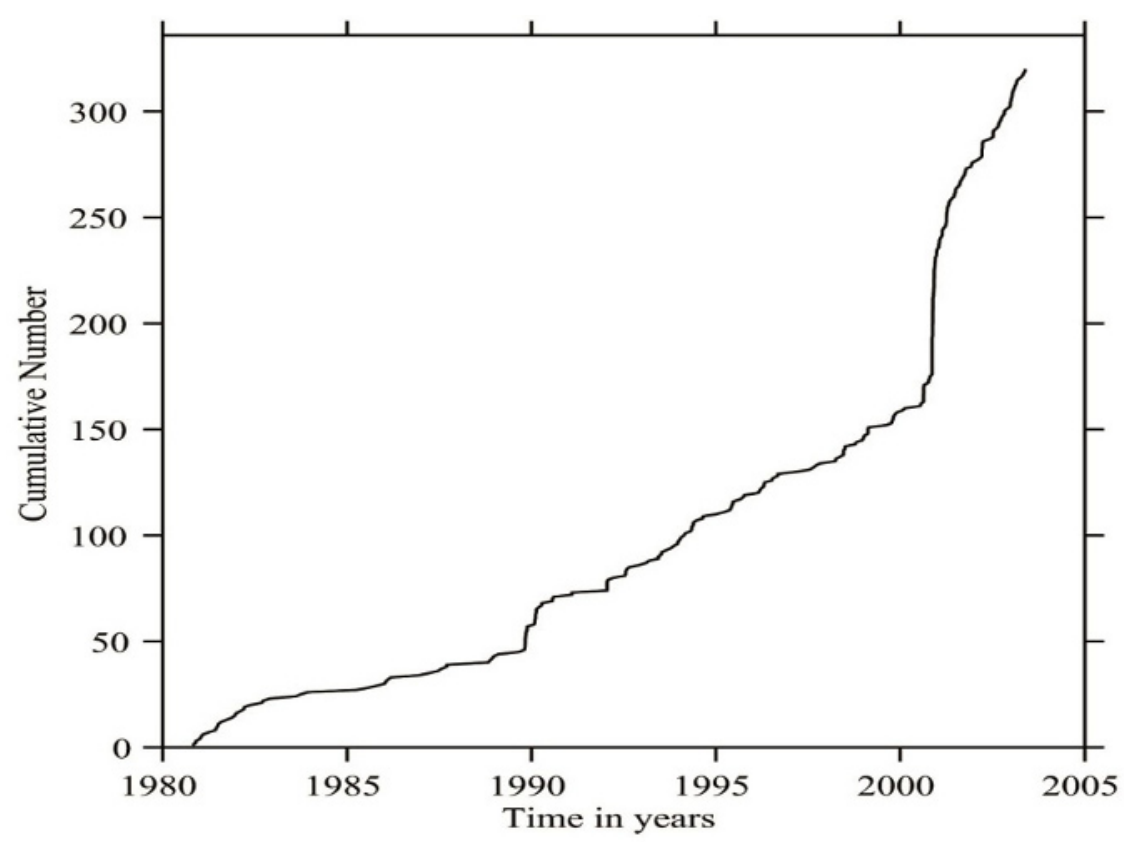

Fig. A1. Cumulative number of earthquakes with magnitude $M s \geq 2$ occured in the study region during the period 1980-2003.

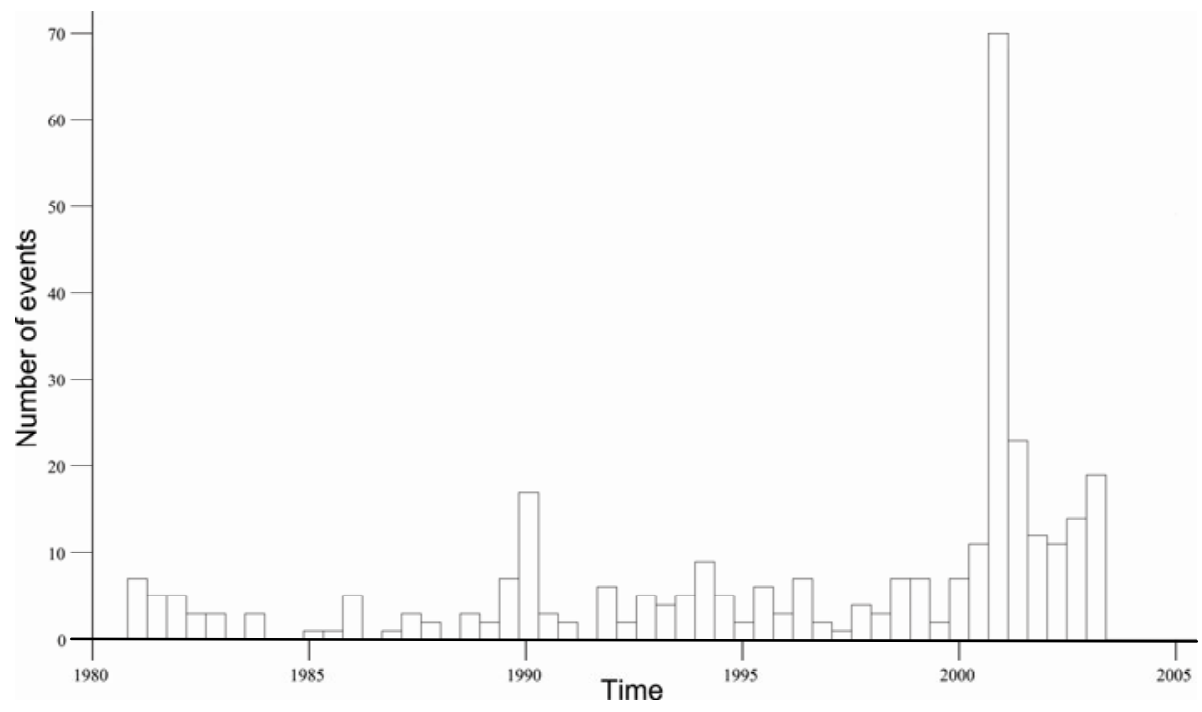

Fig. A2. Histogram of earthquakes with magnitude $M s \geq 2.0$ versus time. 


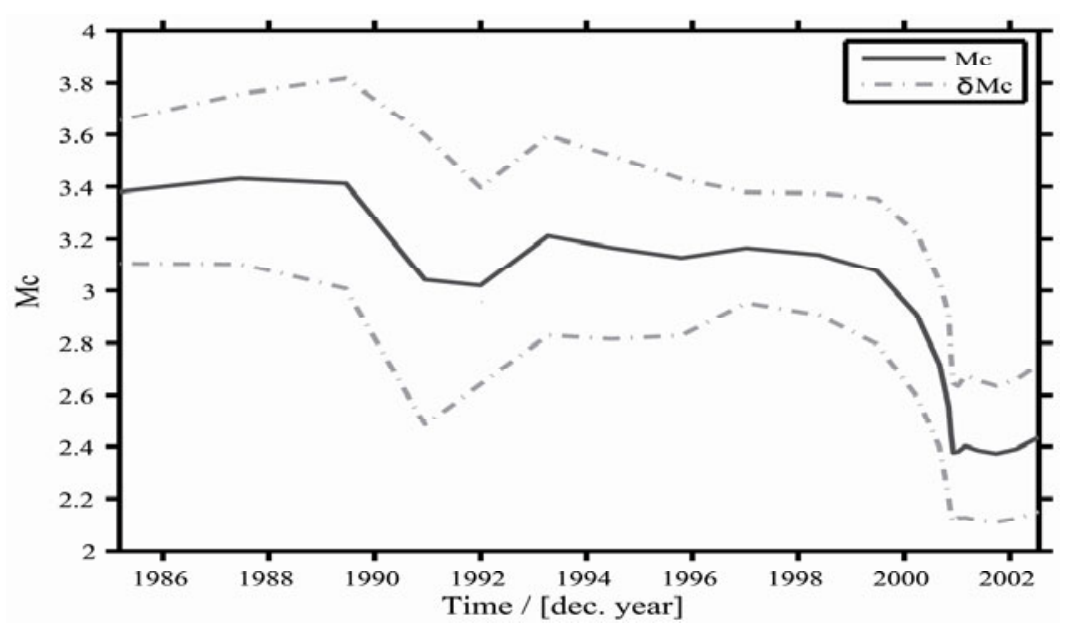

Fig. A3. Variation of magnitude of completeness versus time for the reported seismicity.

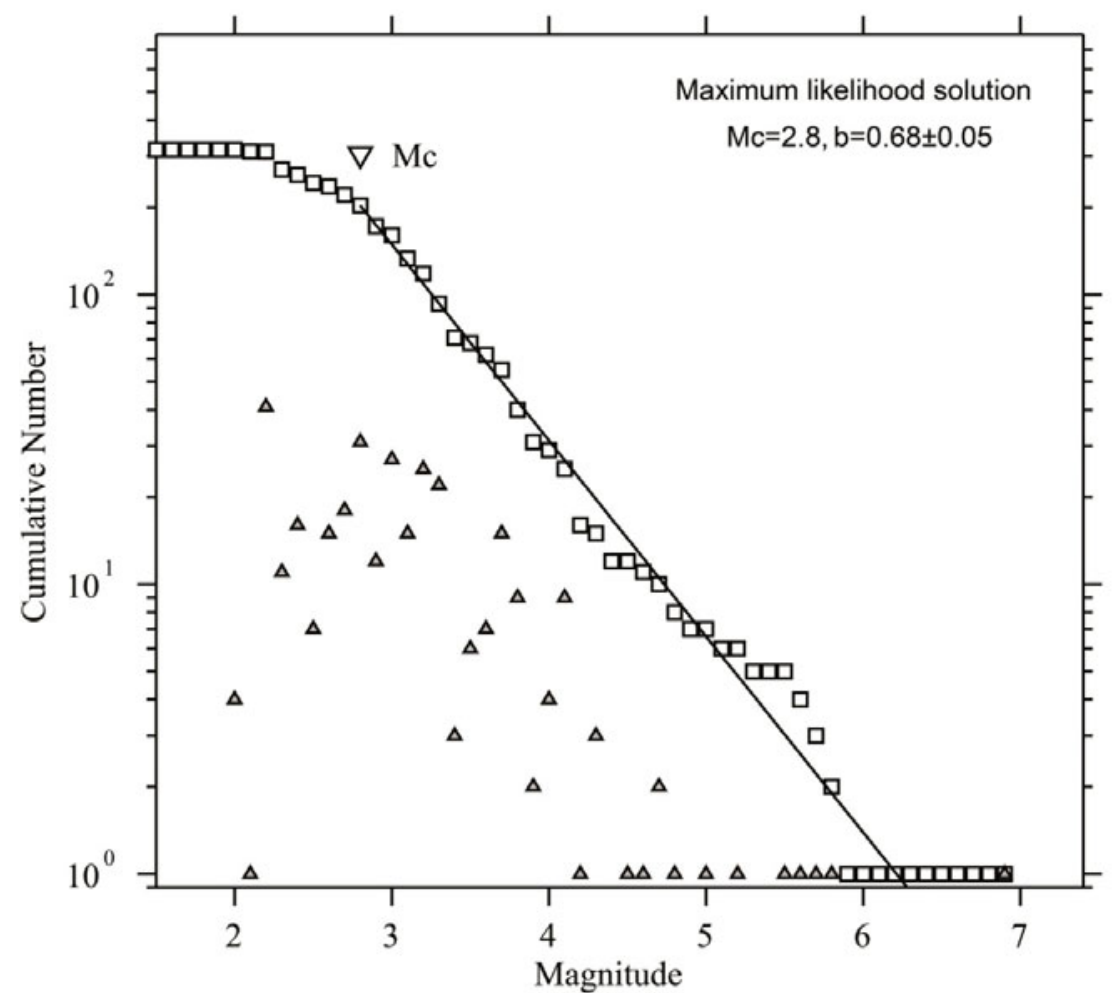

Fig. A4. FMD of the used earthquake catalog. The $b$ value, or slope of the Gutenberg-Richter law, is computed for magnitudes greater than $M c$ (by using the maximum-likelihood technique). 


\section{References}

Aitouche, M.A., M. Djeddi, and K. Baddari (2013), Fractal variogram-based timespace of aftershock sequences analysis-case study: the May 21, 2003 Boumerdes-Algeria earthquake, $\mathrm{M}_{\mathrm{w}}=6.8$, Arab. J. Geosci. 6, 7, 21832192, DOI: $10.1007 / \mathrm{s} 12517-011-0509-y$.

Baddari, K., and A.D. Frolov (1997), Modeling of fractal structure of geophysical field, Cr. Acad. Sci. Sér. Geosci, 325, 12, 925-930.

Baddari, K., and A.D. Frolov (2010), Regularities in discrete hierarchy seismoacoustic mode in a geophysical field, Ann. Geophys. 53, 5-6, 31-42, DOI: 10.4401/ag.4725.

Baddari, K., G.A. Sobolev, and A.D. Frolov (1996), Similarity in seismic precursors at different scales, Cr. Acad. Sci. Sér. Geosci. 323, 9, 755-763.

Baddari, K., G.A. Sobolev, A.D. Frolov, and A.V. Ponomarev (1999), An integrated study of physical precursors of failure in relation to earthquake prediction using large scale rock blocks, Ann. Geofis. 42, 5, 771-787.

Baddari, K., A.D. Frolov, V. Tourtchine, and F. Rahmoune (2011), An integrated study of the dynamics of electromagnetic and acoustic regimes during failure of complex macrosystems using rock blocks, Rock Mech. Rock Eng. 44, 3, 269-280, DOI: 10.1007/s00603-010-0130-5.

Baddari, K., A.D. Frolov, V. Tourtchine, S. Makdeche, and F. Rahmoune (2012), Effect of temperature on the physical precursors of rock block failure, Acta Geophys. 60, 4, 1007-1029, DOI: 10.2478/s11600-012-0038-4.

Baddari, K., S. Makdeche, and F. Bellelem (2013), Probabilistic model to forecast earthquakes in the Zemmouri (Algeria) seismoactive area on the basis of moment magnitude scale distribution functions, Acta Geophys. 61, 1, 6083, DOI: $10.2478 / \mathrm{s} 11600-012-0064-2$.

Baddari, K., A.D. Frolov, V. Tourtchine, F. Rahmoune, and S. Makdeche (2015), Effect of stress-strain conditions on physical precursors and failure stages development in rock samples, Acta Geophys. 63, 1, 62-102, DOI: 10.2478/ s11600-014-0206-9.

Benouar, D. (1994), Materials of investigations of the seismicity of Algeria and adjacent regions, Ann.Geofis. 37, 4, 853-860, DOI: 10.4401/ag-4466.

Caneva, A., and V. Smirnov (2004), Using the fractal dimension of earthquake distributions and slope of the recurrence curve to forecast earthquakes in Colombia, Earth Sci. Res. J. 8, 1, 3-9.

Console, R., M. Murri, and A.M. Lombardi (2003), Refining earthquake clustering models, J. Geophys. Res. 108, B10, 2468, DOI: 10.1029/2002JB002130.

Console, R., M. Murru, F. Catalli, and G. Falcone (2007), Real time forecasts through an earthquake clustering model constrained by the rate- andstate constituve law: comparison with a purely stochastic ETAS model, Seismol. Res. Lett. 78, 1, 49-56, DOI: 10.1785/gssrl.78.1.49. 
Crownover, R.M. (1995), Introduction to Fractals and Chaos, Jones and Bartlett Pub. Inc., Boston-London, 195 pp.

Csikor, F.F., C.R. Motz, D. Weygand, M. Zaiser, and S. Zapperi (2007), Dislocation avalanches, strain bursts, and the problem of the plastic forming at the micrometer scale, Science 318, 5848, 251-254, DOI: 10.1126/science. 1143719.

Dahmen, K.A., Y. Ben-Zion, and J.T. Uhl (2009), Micromechanical model for deformation in solids with universal predictions for stress-strain curves and slip avalanches, Phys. Rev. Lett. 102, 17, 175501, DOI: 10.1103/ PhysRevLett.102.175501.

Das, R., H.R. Wason, and M.L. Sharma (2011), Global regression relations for conversion of surface wave and body wave magnitudes to moment magnitude, Nat. Hazards 59, 2, 801-810, DOI: 10.1007/s11069-011-9796-6.

De Haan, L. (1990), Fighting the arch-enemy with mathematics, Stat. Neerl. 44, 2 , 45-68, DOI: 10.1111/j.1467-9574.1990.tb01526.x.

Evison, F. (1999), On the existence of earthquake precursors, Ann. Geofis. 42, 5, 763-770.

Fukao, Y., and M. Furumento (1985), Hierarchy in earthquake size distribution, Phys. Earth Planet. In. 37, 149-168.

Gardner, J.K., and L. Knopoff (1974), Is the sequence of earthquakes in southern California, with aftershocks removed, Poissonian? Bull. Seismol. Soc. Am. 64, 5, 1363-1367.

Gusev, A.A. (1991), Intermagnitude relationship and asperity statistics, Pure Appl. Geophys. 136, 4, 515-527, DOI: 10.1007/BF00878585.

Huber, P.J., and E.M. Ronchetti (2011), Robust Statistics, 2nd ed., John Wiley \& Sons Inc., New York, 380 pp.

Hurst, H.E. (1965), Long-term Storage: An Experimental Study, Constable, London.

Kanamori, H. (1977), The energy release in great earthquake, J. Geophys. Res. 82, 20, 2981-2987, DOI: 10.1029/JB082i020p02981.

Kanamori, H. (2004), The density of the physics of earthquakes, Proc. Jap. Acad. B 80, 7, 297-316.

Kasahara, K. (1985), Earthquake Mechanism, MIR, Moscow, 264 pp.

Kendall, M., and A. Stuart (1961), The Advanced Theory of Statistics. Vol. 2. Inference and Relationship, Hafner, New York.

Kolmogorov, A.N. (1974), The Basic Concepts of Probability Theory, Nauka, Moscow, 200 pp. (in Russian).

Lavanda, B.H., and E. Cipollone (2000), Extreme values statistics and thermodynamics of earthquakes: aftershocks sequences, Ann. Geofis. 43, 5, 967-982.

Main, I.G., P.G. Meridith, P.R. Sammonds, and C. Jones (1990), Influence of fractal flaw distributions on rock deformation in the brittle field, Geol. Soc. London Sp. Publ. 54, 81-96, DOI: 10.1144/GSL.SP.1990.054.01.09. 
Mokrane, A., A. Ait Messaoud, A. Sebai, A. Ayadi, M. Bezzeghoud, and H. Benhallou (1994), Les séismes en Algérie de 1365 à 1992, Publication du Centrede Recherche en Astronomie, Astrophysique et Géophysique. Département: Etudes et Surveillance Sismique, ESS, CRAAG, Alger-Bouzaréah (in French).

Ogata, Y., K. Katsura, and M. Tanemura (2003), Modelling heterogeneous spacetime occurrences of earthquakes and its residual analysis, J. Roy. Stat. Soc. C 52, 4, 499-509, DOI: 10.1111/1467-9876.00420.

Potanina, M.G., V.B. Smirnov, A.V. Ponomarev, P. Bernard, A.A. Lyubushinb, and Sh.P. Shoziyoev (2015), The pattern of acoustic emission under fluid initiation of failure: laboratory modeling, Izv. Phys. Solid Earth 51, 2, 278-289, DOI: $10.1134 / \mathrm{S} 1069351315020068$.

Riznichenko, Yu.V. (1976), Crustal earthquake source size and the seismic moment, In: Yu.V. Riznichenko (ed.), Earthquake Physics Studies, Nauka, Moscow, 9-27.

Sadovsky, M.A., L.G. Bolkhovitinov, and V.F. Pisarenko (1991), Deformation of the Geophysical Medium and Seismic Process, Nauka, Moscow, 100 pp. (in Russian).

Scholz, C.H. (2010), Large earthquake triggering, clustering, and the synchronization of faults, Bull. Seismol. Soc. Am. 100, 3, 901-909, DOI: 10.1785/ 0120090309.

Shi, Y., and B.A. Bolt (1982), The standard error of the magnitude frequency b-value, Bull. Seismol. Soc. Am. 72, 5, 1677-1687.

Smirnov, V.B., A.V. Ponomarev, and A.D. Zavyalov (1995), Acoustic structure in rock samples and the seismic process, Izv. Phys. Solid Earth 31, 1, 38-58.

Smirnov, V.B., R.K. Chadha, A.V. Ponomarev, and D. Srinagesh (2013), Prognostic anomalies of induced seismicity in the region of the Koyna-Warna wtar reservoirs, west India, Izv. Phys. Solid Earth 49, 2, 243-257, DOI: 10.1134/ S1069351313020080.

Sobolev, G.A. (1995), Fundamental of Earthquake Prediction, ERC, Moscow, $161 \mathrm{pp}$.

Sobolev, G.A. (2011), Seismicity dynamics and earthquake predictability, Nat. Haz. Earth Syst. Sci. 11, 2, 445-458, DOI: 10.5194/nhess-11-445-2011.

Sobolev, G.A., and A.V. Ponomarev (2003), Earthquake Physics and Precursors, Nauka, Moscow, 270 pp. (in Russian).

Utsu, T. (2002), Relationships between magnitude scales. In: W.H.K. Lee, H. Kanamori, P.C. Jennings, and C. Kisslinger (eds.), International Handbook of Earthquake and Engineering Seismology. Part A, Academic Press, Amsterdam, 733-746.

Woessner, J., and S. Wiemer (2005), Assessing the quality of earthquake catalogues: Estimating the magnitude of completeness and its uncertainty, Bull. Seismol. Soc. Am. 95, 2, 684-698, DOI: 10.1785/0120040007. 
Yelles Chaouche, A.K., A. Deramchi, A. Ferkoul, and K. Aoulaiche (2002), Les séismes en Algérie de 1992 à 2001, Publication du Centre de Recherche en Astronomie, Astrophysique et Géophysique. Département: Etudes et Surveillance Sismique, ESS, CRAAG, Alger-Bouzaréah (in French).

Zavyalov, A.D. (2006), Intermediate Term Earthquake Prediction, Nauka, Moscow, 254 pp. (in Russian).

Zhuang, J., Y. Ogata, and D. Vere-Jones (2004), Analyzing earthquake clustering features by using stochastic reconstruction. J. Geophys. Res. 109, B5, B05301, DOI: 10.1029/2003JB002879.

Received 30 June 2015

Received in revised form 9 October 2015

Accepted 29 October 2015 